\title{
A new method for choosing between ball-end cutter and toroidal cutter when machining free-form surfaces
}

\author{
Mahfoud Herraz ${ }^{\mathrm{a}}$, Jean-Max Redonneta ${ }^{\mathrm{a}}$, Marcel Mongead ${ }^{\mathrm{b}}$ and Mohammed SBini ${ }^{\mathrm{b}}$ \\ ${ }^{a}$ Université Paul Sabatier and Institut Clément Ader, Université de Toulouse, France \\ b ENAC, Université de Toulouse, France
}

The International Journal of Advanced Manufacturing Technology, vol. 111 (5), pp 1425-1443, 2020

DOI: $10.1007 / \mathrm{s} 00170-020-06087-\mathrm{y}$

\begin{abstract}
End-milling of free-form surfaces on multi-axis CNC machines are complex and expensive operations involved in the production of many high-value parts, molds and stamping dies. For such operations, the choice of the cutter type to use is very important given the considerable impact of this choice on the quality of the machined surface and the duration of the operation. In this paper, a new method for choosing between ball-end cutter and toroidal cutter is provided. This procedure gives a good hint on the best tool to employ with no need to carry out any machining simulation.
\end{abstract}

Keywords: end milling; free-form surface; ball-end cutter; toroidal cutter; principal component analysis

\section{Contents}

\section{Introduction}

2 Material methods

2.1 Cutter types and effective radius calculation . .

2.2 Kinematics concerns . . . . . . . . . . . .

3 Problem statement

4 Study of a plane surface

4.1 Analytical study . . . . . . . . . . . . 5

4.2 Numerical validation . . . . . . . . . . . . . 8

5 Extension to free-form surfaces

5.1 Preliminary considerations . . . . . . . . . 9

5.2 Using the covariance matrix to define the principal direction . . . . . . . . . . . . 10

5.3 Using eigenvalues to define an approximate form factor of a free-from surface . . . . . . . . 10

5.4 Approximated slope of a free-form surface . . . 11

6 Practical application to free-form surfaces 6.1 Step-by-step description of the method to choose the best cutter type . . . . . . . . . 11

6.2 Test cases . . . . . . . . . . . . . . . 11

7 Discussion 13

7.1 On the efficiency of the proposed method ... 14

7.2 Limitations of scope . . . . . . . . . . . . . 14
7.3 On the extension to free-form surfaces . . . . 14

7.4 Influence of auxiliary parameters . . . . . . 15

8 Conclusion and perspectives $\quad 15$

$\begin{array}{ll}\text { Appendices } & 17\end{array}$

A Study of the critical parameter $\mu_{c}=f(s) \quad \mathbf{1 7}$

A.1 Analytical expression of $\mu_{c} \ldots \ldots . . . .17$

A.2 Numerical analysis of the critical parameter $\mu_{c} \quad 17$

B Proof that form-factor approximation by eigenvalues is exact for a plane

C Proof that the critical functions are decreasing 18

D Proof that the critical slope increases with $\chi$ 


\section{Introduction}

The quality constraint of the surface is commonly expressed in terms of maximum scallop height, denoted $s h$, which corresponds to the residual stock thickness left unmachined by the tool between two adjacent trajectories. This value imposes the step-over distance sod that can be used during the milling of the surface. Roughly speaking, the step-over distance is the distance between two adjacent trajectories. The relation between $s h$ and $s o d$ is well known and fully developed in [1]. The step-over distance is a key parameter for end-milling of free-form surfaces because, for a given scallop height, a greater sod leads to fewer trajectories and thus a reduced machining time.

Numerous authors have addressed these issues [2-6]. Most of them use the concepts of effective radius and sweep curve to do so. The sweep curve is the curve lying on the spinning cutter envelope surface, that defines the final profile of the cutter passage $[7,8]$. From a kinematics point of view, the sweep curve is given by $\mathbf{n} \cdot \mathbf{F}=0$, where $\mathbf{F}$ is the feed direction and $\mathbf{n}$ the normal vector that can be calculated at each point of the cutter surface of revolution. Then, for a given cutter, the effective radius (denoted $R_{\text {eff }}$ ) is defined as the radius of curvature at the cutter contact point of the projection, in a plane normal to the feed direction, of the sweep curve. The direct impact of the effective radius on machining time is thus well established [9]. However, effective radius calculation may indeed vary a lot depending on the cutter geometry in use.

The remaining of this paper is organized as follows. Section 2 recalls $R_{\text {eff }}$ calculation methods, depending on the cutter shape. For each cutter type, kinematics considerations are also reminded in order to explain how machining time can be calculated. Section 3 is dedicated to the detailed problem presentation, illustrated with examples. The next two sections deals respectively with the simple case of an inclined plane, and with an extension to free-form surfaces. More preceisely, Section 4 aims to highlight some critical parameters to be the decisive factors in the choice of a cutter type, while Section 5 focuses on the best way to interpret these parameters in the context of free-form surfaces. Section 6 is dedicated to a practical application to free-form surfaces: a step-bystep description of the method we propose is presented, and then illustrated by two test cases carried out in a practical context. Remark that full understanding of the above theoretical developments is not required to follow the application section. Finally, the pros and cons of our approach are summarized and discussed in Section 7. Particularly, this method is shown to be useful for the problematic cases for which the choice of a cutter type is not obvious.

\section{Material methods}

\subsection{Cutter types and effective radius cal- culation}

End-milling of free-form surfaces can be performed with various kind of tools. Actually three types are commonly used (Figure 1):

- the flat-end cutter, which is less used because of the sharp marks it leaves on the part surface

- the spherical, or ball-end, cutter, which is the most commonly used in industry, because using it toolpaths are easy to calculate

- the toroidal cutter, which have been proven to be capable results better than those of ball-end cutter, although it is much harder to handle in calculations.

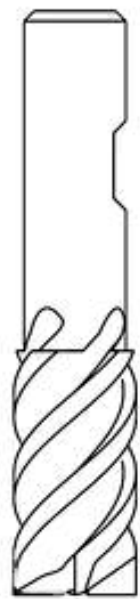

flat-end mill

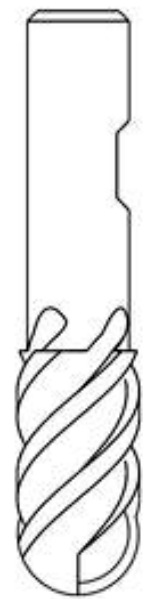

ball-end mill

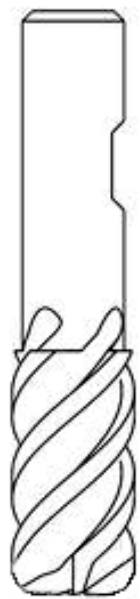

toroidal mill
Figure 1: The three main tool types used in end-milling

An important parameter for choosing cutter geometry is the effective radius. This notion was initially introduced in [10]. For a practical usage, the effective radius $R_{\text {eff }}$ can be calculated as follows:

- flat-end cutter: its effective radius depends on the tilt angle $\phi$. As defined in [10]: $R_{\text {eff }}=\frac{R}{\sin \phi}$, where $R$ is the tool radius

- ball-end cutter: its effective radius is equal to the tool radius regardless of the tool orientation: $R_{\text {eff }}=$ $R$

- toroidal cutter: its effective radius depends on the tool radius $R$, the torus radius $r$, the steepest-slope $s$, and the angle between the machining direction and 
the steepest-slope direction $\alpha$. Equation 1 proposed by [1] details the expression of $R_{\text {eff }}$ :

$$
R_{e f f}=\frac{(R-r) \cos ^{2} \alpha}{\sin s\left(1-\sin ^{2} \alpha \sin ^{2} s\right)}+r
$$

The spherical tool is widely used in industry because it does not require calculation of the effective radius, which makes the generation of machining paths quite simple. In addition, it leaves smoother marks on the surface than a flat-end tool, which results in less roughness. However, the flat-end cutter allows a greater effective radius than its ball-end tool counterpart. The toroidal cutter, as shown in [11], inherits the merits of both previouslymentionned cutters. Actually, it may allow, depending on the machining direction and surface geometric characteristics, greater effective radius than the spherical tool, while leaving traces on the surface smoother than those of a same-radius flat-end cutter.

Nowadays flat-end mills are, so to speak, no longer used for finishing free-form surfaces because of the pronounced marks they left on the surface, leading to a high roughness in the feed direction $[12,13]$. Therefore, they are not taken into consideration in the present study.

Figure 2 shows step-over distances allowed by ball-end cutter (above) and toroidal cutter (below), when machining in the steepest-slope direction (blue cutter) or in a direction perpendicular to this one (green cutter). All the scallop heights are the same across the whole figure. A simple plane have been chosen as surface to make the figure easier to read, but the same results are obtained for free-form surfaces.

On this figure, one can see that for the ball-end mill, the effective radius, and thus the step-over distance, is the same whatever the milling direction chosen. It is also clear that the use of a toroidal mill in the steepest-slope direction leads to better results than the use of a ball-end mill in the same conditions, while the use of a toroidal cutter in the direction perpendicular to the steepest-slope direction leads to worse results than those obtained using a ball-end mill in the same conditions.

It has been proven in [14] that the effective radius of a toroidal cutter of radii $R$ and $r$ is superior to that obtained by a spherical tool of radius $R$, when the angle $\alpha$ is within the range $\left[-35^{\circ}, 35^{\circ}\right]$. This condition is sufficient regardless of the values of $R, r$ and $s$.

Once the effective radius known, the scallop height is quite easy to calculate. For toroidal cutters, using Equation (1) allows calculation much faster than numerical methods previously employed.

\subsection{Kinematics concerns}

The production cost of a surface is directly linked to the machining time. Many authors, for example [15-21], assume that the machining time is proportional to the
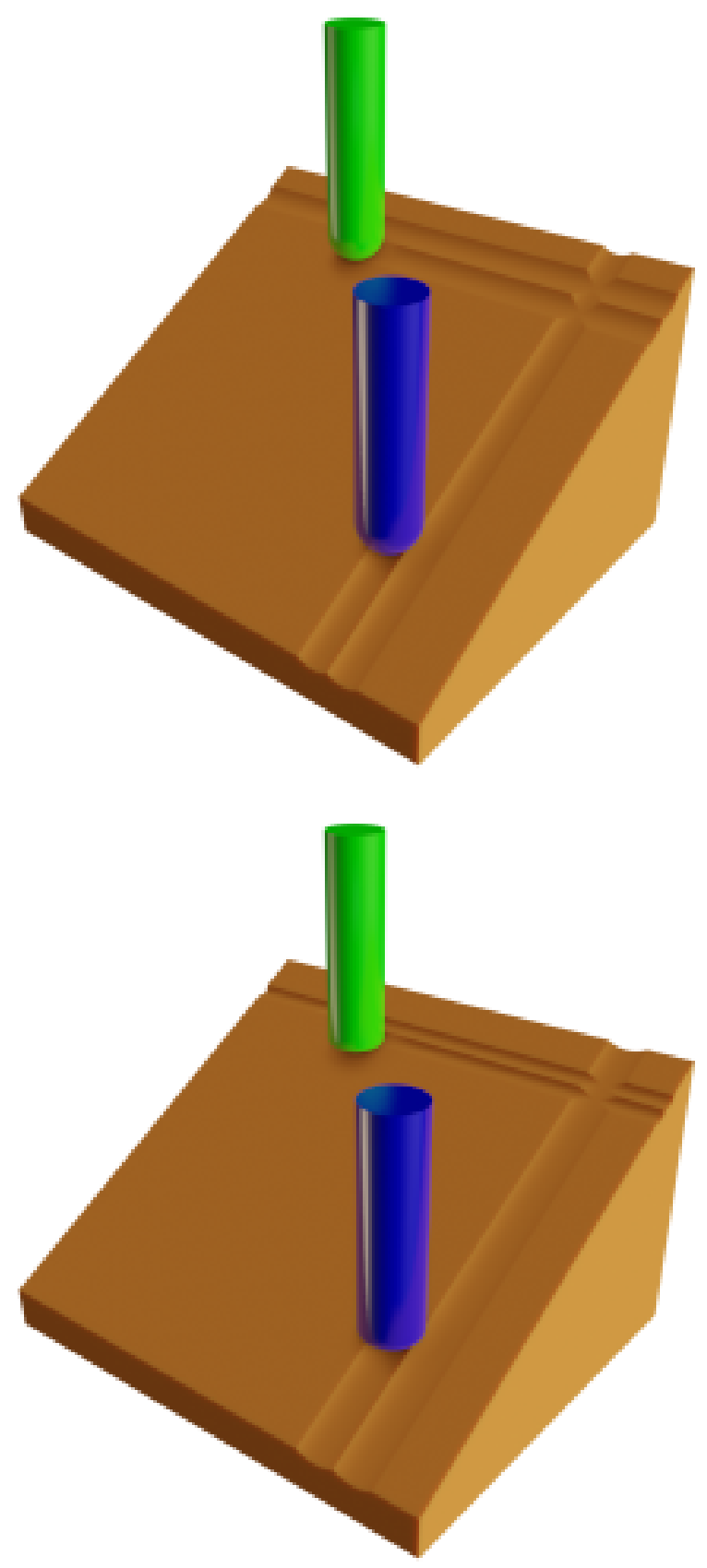

Figure 2: Ball-end mill (above) versus toroidal cutter (below) regarding surface slope

length of the toolpath, and thus focus on minimizing the toolpath length, rather than the machining time. This approximation is not exact unless the speed of the tool is constant, which is ideal but pretty much not realistic. In practice, the cutter must decelerate when arriving to an acute angle (i.e. a linear path with tangency discontinuity). Likewise, it has to accelerate once this angle passed. Obviously the cutter cannot pass through a tangency discontinuity without lowering its speed to zero. To avoid a complete zero speed, current numerical controllers (NC) 
allow a curved path within a given tolerance, but this tolerance is kept small enough to prevent having a visible impact on the real path. Thus, the feed speed still has to decrease approaching sharp angles, and then to increase back once this angle passed. Further explanation (and optimization) of this problem can be found in [22].

Therefore, the kinematic capabilities of the CNC machine should be considered since the tool displacement characteristics must be kept within these capabilities (see [23] for a full modelling of 3-axis machines kinematics).

In order to calculate the time needed to go through a linear path portion between two accurate angles, some values need to be known:

- the nominal feed rate, denoted $V(\mathrm{~m} / \mathrm{s})$

- the maximum acceleration, denoted $A_{\max }\left(\mathrm{m} / \mathrm{s}^{2}\right)$

- the maximum gradient of acceleration, also called jerk, denoted $J_{\max }\left(\mathrm{m} / \mathrm{s}^{3}\right)$

Using these notations, three cases may arise:

1. the nominal feed rate $V$ and the maximum acceleration $A_{\max }$ are achieved

2. the nominal feed rate $V$ is achieved, but not the maximum acceleration $A_{\max }$

3 . the nominal feed rate $V$ and the maximum acceleration $A_{\max }$ are not achieved.

For a line segment of length $2 l$, according to the values of $V, A_{\max }$ and $J_{\max }$, the following characteristic times are also defined:

- the time to reach the maximum acceleration in case 1: $t_{A}=\frac{A_{\max }}{J_{\max }}$

- the time to reach the nominal feed rate in case 2 : $t_{V}=\sqrt{\frac{V}{J_{\max }}}$

- the time to travel the half length distance $l$ in case 3 : $t_{l}=\sqrt[3]{\frac{l}{J_{\max }}}$

These characteristic times help to distinguish between the previously-stated cases. The characterization of each case and the corresponding machining time $t$ are then as follows:

- case 1: if $t_{A}<t_{V}$ and $t_{A}<t_{l}$,

$$
t=2 \frac{l}{V}+\frac{A_{\max }}{J_{\max }}+\frac{V}{A_{\max }}
$$

- case 2: if $t_{V}<t_{A}$ and $t_{V}<t_{l}$,

$$
t=2 \frac{l}{V}+2 \sqrt{\frac{V}{J_{\max }}}
$$

- case 3: if $t_{l}<t_{A}$ and $t_{l}<t_{V}$,

$$
t=4 \sqrt[3]{\frac{l}{J_{\max }}}
$$

In particular, when $t_{V}<t_{A}$, which will be the most common case for the kinematic parameters values used in this paper, the case 1 is dismissed and the case 2 (respectively the case 3 ) applies if and only if $l>\sqrt{\frac{V^{3}}{J_{\max }}}$ (respectively $l<\sqrt{\frac{V^{3}}{J_{\max }}}$ ).

Hence, the machining time associated with a toolpath increases when the number of acute angles increases, eventhough the path length is fixed.

\section{Problem statement}

As it was previously established, using the toroidal cutter along the steepest-slope direction maximizes the effective radius over the whole surface, which leads to larger step-over distances sod between adjacent paths, reducing thereby the total toolpath length. However, if the steepest-slope direction is near the perpendicular to the direction of the surface's largest dimension, then the generated toolpath will be composed of shorter paths. In this case, using a ball-end cutter along the direction of the surface's largest dimension, hereafter called principal direction, may provide better results in terms of machining time, despite a longer toolpath. Indeed, the kinematic behaviour of the tool, that needs to decelerate nearing each path angle and then accelerate again once the path angle passed, is much more penalizing in the first case than in the second one.

In order to illustrate this issue, let us consider a rectangular plane surface of width $w=56 \mathrm{~mm}$ and height $h=28 \mathrm{~mm}$; its slope angle is $60^{\circ}$ and the steepestslope direction is perpendicular to the principal direction (Figure 3). This plane surface is first machined using a toroidal cutter of radii $R=5 \mathrm{~mm}$ and $r=2 \mathrm{~mm}$ along the steepest-slope direction (lying in the plane $(\mathbf{X}, \mathbf{Z})$ ). Then the same plane is machined using a ball-end cutter of radius $R=5 \mathrm{~mm}$ along the principal direction (axis $\mathbf{Y}$ ). The maximum allowed scallop height is $s h=0.01 \mathrm{~mm}$, which is a standard value in industrial applications.

Results in terms of effective radius, machining time and toolpath length are reported in Table 1 . The toolpath length is the same, yet it is worth noting that even though the effective radius is $9.2 \%$ greater in the first case (machining with a toroidal cutter along the steepest-slope direction), the gain obtained by machining along the principal direction using a ball-end cutter in terms of machining time is nearly $16 \%$.

To deepen the tests, a form factor $\mu$ is defined for the plane such that $\mu=\frac{w}{h}$, where $w$ is the width of the 

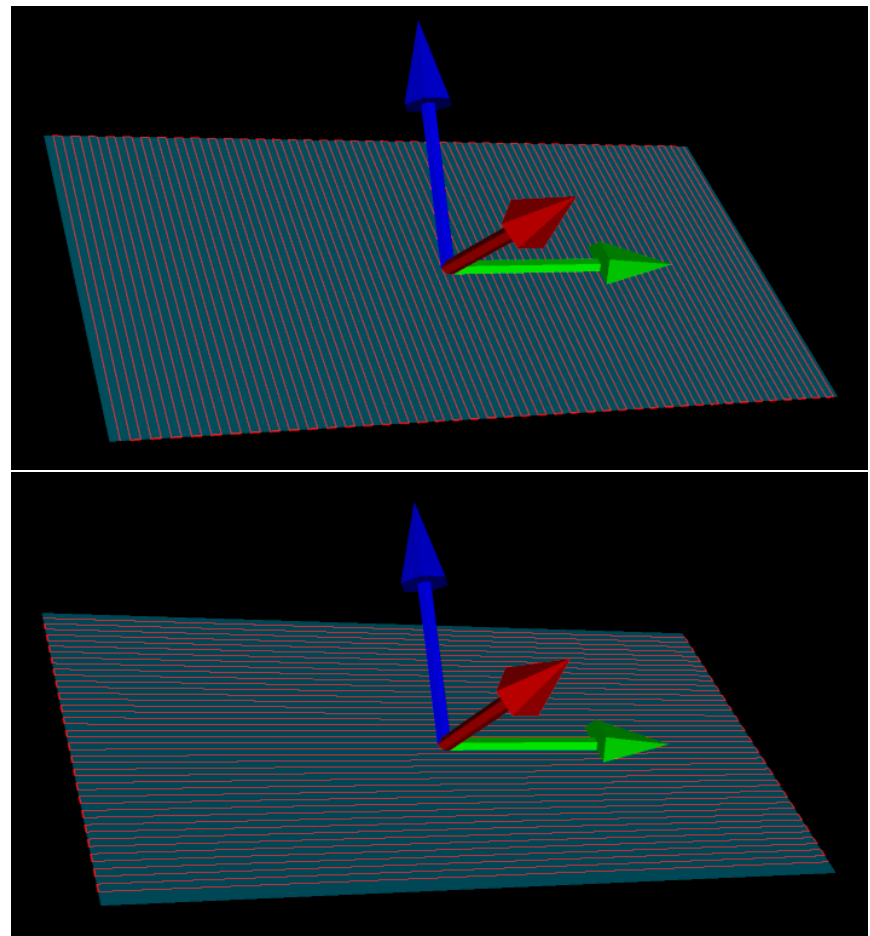

legend: axes $\mathbf{X} / \mathbf{Y} / \mathbf{Z}$ correspond to Red/Green/Blue vectors

Figure 3: Machining of a rectangular planar surface: along the steepest-slope direction using a toroidal cutter (above), along the principal direction using a ball-end cutter (below)

\begin{tabular}{|c|c|c|c|}
\hline $\begin{array}{c}\text { machining } \\
\text { direction }\end{array}$ & $\begin{array}{c}\text { effective } \\
\text { radius }\end{array}$ & $\begin{array}{c}\text { machining } \\
\text { time }\end{array}$ & path length \\
\hline steepest-slope & $5.46 \mathrm{~mm}$ & $43.8 \mathrm{~s}$ & $2460 \mathrm{~mm}$ \\
\hline principal & $5 \mathrm{~mm}$ & $36.8 \mathrm{~s}$ & $2459 \mathrm{~mm}$ \\
\hline
\end{tabular}

Table 1: Results of plane surface machined using toroidal cutter along its steepest-slope direction and ball-end cutter along its principal direction

surface and $h(\leq w)$ is the height. According to this, a characteristic size $\chi$ may be defined such that:

$$
\chi=w h=\frac{w^{2}}{\mu}=h^{2} \mu
$$

Various tests are run for different values of the slope angle $s$ and this form factor $\mu$ for a constant characteristic size. As expected, the toroidal cutter tends to present better machining times when the slope is decreasing towards $0^{\circ}$ and the form factor approaching 1 . Conversely, the spherical cutter tends to be more efficient when the slope is increasing towards $90^{\circ}$ and form factor is high.

In fact, the parameter $\mu$ represents the elongation of the surface, while $\chi$ tells about its size and area. As previously stated, the slope $s$ and the form factor $\mu$ are the most influent parameters on the machining time. Hence, in order to focus on these parameters, the characteristic size $\chi$ is considered constant in this study.

Based on these observations, a well-founded question arises: is it possible to define critical values for the parameters $s$ and $\mu$ that correspond to having equal machining time for both milling processes? If yes, such critical values would correspond to the limit case where both cutters are equally efficient. As a result, for a given practical case, comparing the surface actual values of these parameters with these critical values would permit to predict which tool will be the most efficient. In the following section, this question is investigated for a plane surface.

\section{Study of a plane surface}

The efficiency of the torus tool and the spherical tool are investigated on a rectangular plane surface as the steepest-slope $s$ and the width by length form factor $\mu$ vary. In order to calculate the machining time, the kinematic model of the CNC machine introduced in Section 2.2 is used.

The machining strategy used here is the "zigzag" parallel plans, so that a toolpath is composed of a sequence of linear segments put end to end (i.e., NC code: G01). In the sequel, the kinematic parameters are fixed to: $J_{\max }=40 \mathrm{~m} / \mathrm{s}^{3}, A_{\max }=6 \mathrm{~m} / \mathrm{s}^{2}, V=5 \mathrm{~m} / \mathrm{min}$, and cutters are defined by outer radius $R=5 \mathrm{~mm}$, and torus radius $r=2 \mathrm{~mm}$ for the toroidal mill. The scallop height constraint is equal to $s h=0.01 \mathrm{~mm}$.

\subsection{Analytical study}

\subsubsection{Toroidal cutter along steepest-slope direc- tion}

In this section, the machining time using a toroidal tool along the steepest-slope direction (upper picture in Figure 3 ) is calculated as a function of the cutter geometry, the kinematic parameters and the surface parameters. For any point of the surface, the effective radius of the toroidal tool can be calculated using Equation 1 with $\alpha=0$ :

$$
R_{e f f}=\frac{R-r}{\sin s}+r .
$$

The step-over distance is given by:

$$
\operatorname{sod}_{1}=2 \sqrt{2 \operatorname{sh} R_{e f f}-s h^{2}}
$$

and therefore the number of paths $n_{1}$ is given by:

$$
n_{1}=\frac{w}{\operatorname{sod}_{1}}=\frac{\sqrt{\mu \chi}}{\operatorname{sod}_{1}} .
$$

The toolpath length is equal to the total length of the paths plus the step-over distances to move from one path 
to the next one:

$$
\begin{aligned}
L_{1} & =n_{1} h=n_{1} \sqrt{\frac{\chi}{\mu}}+n_{1} \operatorname{sod}_{1} \\
& =\frac{\sqrt{\mu \chi}}{\operatorname{sod}_{1}} \sqrt{\frac{\chi}{\mu}}+\frac{\sqrt{\mu \chi}}{\operatorname{sod}_{1}} \operatorname{sod}_{1} \\
& =\frac{\chi}{\sqrt{2 \operatorname{sh} \frac{R-r}{\sin s}+2 \operatorname{shr}-\operatorname{sh}^{2}}}+\sqrt{\mu \chi}
\end{aligned}
$$

To calculate the machining time, the actual kinematic case occurring must be found (see Section 2.2). This depends on the value of $\chi$, since $t_{A}$ et $t_{V}$ are known:

$$
t_{A}=\frac{A_{\max }}{J_{\max }}=\frac{6}{40}=0.15 \mathrm{~s}
$$

and

$$
t_{V}=\sqrt{\frac{V}{J_{\max }}}=\sqrt{\frac{5}{60 \times 40}}=0.045 \mathrm{~s} .
$$

Since $t_{V}<t_{A}$, the case 1 is dismissed, and the case 2 applies if $t_{V}<t_{l}$. Considering $t_{l}=\sqrt[3]{\frac{l}{J_{\max }}}$ with $l=\frac{h}{2}=$ $\frac{1}{2} \sqrt{\frac{\chi}{\mu}}$, this last condition is equivalent to

$$
\frac{V^{3}}{J_{\max }^{3}}<\frac{\chi}{4 \mu J_{\max }^{2}}
$$

whence

$$
h=\sqrt{\frac{\chi}{\mu}}>2 \sqrt{\frac{V^{3}}{J_{\max }}}=7.6 \mathrm{~mm} .
$$

In the same way, since $t_{V}<t_{A}$, the case 3 is equivalent to $t_{l}<t_{V}$, thus $h<7.6 \mathrm{~mm}$. This value can be considered too small for machinable surfaces. Therefore, in the following, only the case 2 will be taken into consideration. Hence, the machining time for a single path is (see Equation (3)):

$$
t_{p}=\frac{h}{V}+2 \sqrt{\frac{V}{J_{\max }}}=\frac{\sqrt{\chi}}{\sqrt{\mu} V}+2 \sqrt{\frac{V}{J_{\max }}} .
$$

The machining time necessary to move from one path to the next needs to be calculated too. It depends on the step-over distance. The kinematics of the machine corresponds to the case 3 if $\operatorname{sod}_{1}<7.6 \mathrm{~mm}$, which is always true since in the worst case $\left(s=0^{\circ}\right)$ one had $\operatorname{sod}_{1}=2(R-r)+2 \sqrt{r^{2}-(r-s h)^{2}}=6.4 \mathrm{~mm}$. Therefore the case 3 is assumed for the calculation of the machining time between two adjacent paths (see Equation (4)):

$$
t_{i p}=4 \sqrt[3]{\frac{\operatorname{sod}_{1}}{2 J_{\max }}}
$$

Finally, the total machining time is:

$$
\begin{aligned}
T_{1} & =n_{1}\left(t_{p}+t_{i p}\right) \\
& =\frac{\sqrt{\mu \chi}}{\operatorname{sod}_{1}}\left(\frac{\sqrt{\chi}}{\sqrt{\mu} V}+2 \sqrt{\frac{V}{J_{\max }}}+4 \sqrt[3]{\frac{\operatorname{sod}_{1}}{2 J_{\max }}}\right) .
\end{aligned}
$$

\subsubsection{Ball-end cutter along the principal direc- tion}

In this section the surface is machined with a ball-end cutter along the principal direction (axis $\mathbf{Y}$, green, in Figure 3), which is orthogonal to the steepest-slope direction (axis $\mathbf{X}$, red, in Figure 3), and the machining time is calculated. In this case, the effective radius is $R_{\text {eff }}=R$. It is worth noting that in this case, the toroidal cutter will present a minimal effective radius of $r$ according to this direction. Thus in this case, the ball-end cutter is much more effective than the toroidal cutter.

The step-over distance is written as:

$$
\operatorname{sod}_{2}=2 \sqrt{2 s h R-s h^{2}} .
$$

The number of paths, in this case, is:

$$
n_{2}=\frac{h}{\operatorname{sod}_{2}}=\frac{\sqrt{\chi}}{\sqrt{\mu} \times \operatorname{sod}_{2}}=\frac{\sqrt{\chi}}{2 \sqrt{\mu} \sqrt{2 s h R-s h^{2}}} .
$$

Here again, the toolpath length is equal to the total length of the paths plus the step-over distances to move from one path to the next one:

$$
L_{2}=n_{2}\left(\sqrt{\frac{\chi}{\mu}}+2 \sqrt{2 s h R-s h^{2}}\right) .
$$

Identically to the previous section, the kinematics of the machine is represented by case 2 . Thus, the machining time corresponding to one path is given by:

$$
t_{p}=\frac{\sqrt{\chi \mu}}{V}+2 \sqrt{\frac{V}{J_{\max }}} .
$$

And the machining time to go from the end of one path to the beginning of the next one is

$$
t_{i p}=4 \sqrt[3]{\frac{\operatorname{sod}_{2}}{2 J_{\max }}} .
$$

Finally, the total machining time is:

$$
\begin{aligned}
T_{2} & =n_{2}\left(t_{p}+t_{i p}\right) \\
& =\frac{\sqrt{\chi}}{\sqrt{\mu} \times \operatorname{sod}_{2}}\left(\frac{\sqrt{\chi \mu}}{V}+2 \sqrt{\frac{V}{J_{\max }}}+4 \sqrt[3]{\frac{\operatorname{sod}_{2}}{2 J_{\max }}}\right) .
\end{aligned}
$$

\subsubsection{Critical values of the form factor (width/height ratio) parameter and the slope parameter}

In the previous sections (4.1.1 and 4.1.2), the total toolpath lengths $\left(L_{i}\right)$ and the machining times $\left(T_{i}\right)$ are calculated for both toroidal cutter machining along the steepest-slope direction ( $L_{1}$ and $\left.T_{1}\right)$, and ball-end cutter machining along the principal direction $\left(L_{2}\right.$ and $\left.T_{2}\right)$. This section focuses on comparing the machining time $T_{1}$ 
with its counterpart $T_{2}$. Basically, this comparison is carried out studying the limit case $T_{1}=T_{2}$. Remark that $T_{1}=T_{2}$ is equivalent to:

$$
\begin{aligned}
& \frac{\sqrt{\mu \chi}}{\operatorname{sod}_{1}}\left(\frac{\sqrt{\chi}}{\sqrt{\mu} V}+2 \sqrt{\frac{V}{J_{\max }}}+4 \sqrt[3]{\frac{\operatorname{sod}_{1}}{2 J_{\max }}}\right) \\
& =\frac{\sqrt{\chi}}{\sqrt{\mu} \operatorname{sod}_{2}}\left(\frac{\sqrt{\chi \mu}}{V}+2 \sqrt{\frac{V}{J_{\max }}}+4 \sqrt[3]{\frac{\operatorname{sod}_{2}}{2 J_{\max }}}\right)
\end{aligned}
$$

In the above equation, the kinematic parameters $J_{\max }$, $A_{\max }$ and $V$, as well as the radii $R$ and $r$, are supposed to be known; the step-over distance $\operatorname{sod}_{2}$ is calculated from the cutter radius $R$ and the maximum scallop height $s h$, therefore it is also known; and the characteristic size $\chi$ is considered constant (see Section 3). Therefore, the only influencing parameters are the form factor $\mu$ and the slope $s$ which appears in the expression of the effective radius $R_{\text {eff }}$ used for calculating $\operatorname{sod}_{1}$.

As the form factor $\mu$ increases, the obtained number of paths when using toroidal cutter increases accordingly while its counterpart using spherical cutter remains the same, since the "elongation" takes place in the principal direction, which is orthogonal to the steepest-slope direction. Thus increasing $\mu$ tends to favour machining with a ball-end cutter in the principal direction.

On the other hand, when the slope tends to 0 , the effective radius of the toroidal cutter tends to infinity which maximizes the transverse pitch and favour machining with a toroidal cutter in the direction of the steepestslope.

In order to determine which cutter geometry and machining direction are best, an analysis of these influencing parameters needs to be carried out. This can be done either by expressing the slope $s$ as a function of the form factor $\mu$, or by expressing the form factor $\mu$ as a function of the slope $s$. From a practical point of view, it may be more useful to express the slope $s$ as a function of the form factor $\mu$. Indeed, for a given geometry the form factor is already defined, while the slope $s$ may still be modified, by setting up the part orientation in the machine-tool workspace. Therefore, the expression of the slope $s$ as a function of the form factor $\mu$ is presented hereafter while the expression of the form-factor $\mu$ as a function of the slope $s$ is presented in Appendix A.1.

Working on the expression of the slope $s$ as a function of the form-factor $\mu$, aims to bring up a critical value of $s$, hereafter called $s_{c}$, that differentiate apart the case for which machining with a toroidal cutter leads to better results than its counterpart. For a given form factor $\mu$, the critical slope $s_{c}$ is defined so that the machining times
$T_{1}$ and $T_{2}$ are equal. Again, $T_{1}=T_{2}$ is equivalent to:

$$
\begin{aligned}
& \frac{\sqrt{\mu \chi}}{\operatorname{sod}_{1}}\left(\frac{\sqrt{\chi}}{\sqrt{\mu} V}+2 \sqrt{\frac{V}{J_{\max }}}\right.\left.+4 \sqrt[3]{\frac{\operatorname{sod}_{1}}{2 J_{\max }}}\right)=T_{2}, \\
& \text { or } \frac{T_{2}}{\sqrt{\mu \chi}} \operatorname{sod}_{1}-\frac{4}{\sqrt[3]{2 J_{\max }}} \sqrt[3]{\operatorname{sod}_{1}} \\
&-\frac{\sqrt{\chi}}{\sqrt{\mu} V}-2 \sqrt{\frac{V}{J_{\max }}}=0 .
\end{aligned}
$$

The last equation is a third-degree polynomial in $z=$ $\sqrt[3]{\operatorname{sod}_{1}}$ of the form:

$$
z^{3}-p z-q=0
$$

where:

$$
\begin{gathered}
p=\frac{4 \sqrt{\mu \chi}}{T_{2} \sqrt[3]{2 J_{\max }}}, \text { and } \\
q=\frac{\sqrt{\mu \chi}}{T_{2}}\left(\frac{\sqrt{\chi}}{\sqrt{\mu} V}+2 \sqrt{\frac{V}{J_{\max }}}\right)
\end{gathered}
$$

It is straightforward to see that $p$ and $q$ are positive. Cardano's formula gives the general solution for Equation (10). The discriminant of this equation is:

$$
\begin{aligned}
\Delta & =4 p^{3}-27 q^{2} \\
& =\frac{\mu \chi}{T_{2}^{2}}\left(128 \frac{\sqrt{\mu \chi}}{J_{\max } T_{2}}-27\left(\frac{\sqrt{\chi}}{\sqrt{\mu} V}+2 \sqrt{\frac{V}{J_{\max }}}\right)^{2}\right) .
\end{aligned}
$$

Using numerical values of $V$ and $J_{\max }$, limiting the width by height form factor to 9 (which means $\sqrt{\mu}<3$ ), and assuming big enough surfaces so that the machining times order of magnitude is superior to one second $\left(T_{2}>\right.$ $1 \mathrm{~s})$, it comes that:

$$
\Delta=\frac{\mu \chi}{T_{2}^{2}}\left(3.2 \frac{\sqrt{\mu \chi}}{T_{2}}-27\left(12 \frac{\sqrt{\chi}}{\sqrt{\mu}}+0.1\right)^{2}\right)
$$

Thus,

$$
\Delta<\frac{\mu \chi}{T_{2}^{2}}\left(9.6 \sqrt{\chi}-27(4 \sqrt{\chi}+0.1)^{2}\right)
$$

The function $\chi \mapsto 9.6 \sqrt{\chi}-27(4 \sqrt{\chi}+0.1)^{2}$ is negative for $\sqrt{\chi}>0.01$. Consequently, $\Delta<0$ for surfaces of area superior to $100 \mathrm{~mm}^{2}$; this hypothesis is fairly acceptable for machinable surfaces. Hence, Equation (10) has one real solution (and two conjugate complex solutions):

$$
z=\sqrt[3]{\frac{-q+\sqrt{\frac{-\Delta}{27}}}{2}}+\sqrt[3]{\frac{-q-\sqrt{\frac{-\Delta}{27}}}{2}} .
$$

Thus, using Equations (6) and (7), the expression of the critical slope $s_{c}(\mu)$ for a given form factor $\mu$ can be 
obtained from

$$
\begin{aligned}
\operatorname{sod}_{1}^{2}=z^{6} & \Leftrightarrow 4\left(2 \operatorname{sh} R_{e f f}-s h^{2}\right)=z^{6} \\
& \Leftrightarrow R_{e f f}=\frac{s h}{2}-\frac{z^{6}}{8 s h}=\frac{R-r}{\sin s_{c}}+r,
\end{aligned}
$$

and finally

$$
s_{c}(\mu)=\arcsin \left(\frac{8 \operatorname{sh}(R-r)}{z^{6}+4 \operatorname{sh}^{2}-8 \operatorname{shr}}\right)
$$

where $\mu$ appears in the $p$ and $q$ components (Equations (11) and (12)) of the value $z$ established in Equation (13).

If $s<s_{c}$ then $\sqrt[3]{\operatorname{sod}_{1}}$ is superior to the solution of Equation (10) and $z^{3}-p z-q>0$, which means that $T_{2}>T_{1}$. In this case, using a toroidal cutter along the steepest-slope direction should be preferred. Conversely, if $s>s_{c}$, then $\sqrt{s o d_{1}}$ is inferior to the solution of equation (10) and $z^{3}-p z-q<0$, which means that $T_{2}<T_{1}$. In this case, ball-end cutter machining along the principal direction is more efficient. This result is consistent with the fact that the effective radius (and step-over distance $\operatorname{sod}_{1}$ ) of toroidal cutter decreases as the slope $s$ increases.

\subsection{Numerical validation}

\subsubsection{Preliminary considerations}

The analytical expression of critical parameter $s_{c}$ is given by Equation (14). This section is devoted to validate this expression using numerical simulations. The machining simulation process used to compute the toolpath for a given parametric surface as a sequence of interpolation points is based on intersection of isoparametric curves and vertical parallel planes. This process is widely tested and effective.

First of all, back to the example given in Section 3, the slope is $s=60^{\circ}$ while the form factor is $\mu=\frac{56}{28}=2$. Using Equation (14), the critical slope can be evaluated: $s_{c}(\mu)=33.8^{\circ}$. Thus $s_{c}<s$, which is coherent with the fact that the machining time using ball-end cutter along the principal direction is shorter. As shown by this example, the proposed procedure is able to provide a good hint for choosing the cutter to use, in order to obtain the best results. Actually, using the critical parameter based procedure, it is possible to predict the tool type leading to shorter machining time without carrying out any machining simulations.

For better understanding of the influence of each parameter, the evolution of the machining times with respect to the form factor $\mu$, for fixed values of $s$, is displayed in Figure 4. Since machining with a ball-end mill does not depend on the slope $s$, one single curve is plotted for the related machining time $\left(T_{2}\right)$. As expected, the machining time $T_{2}$ decreases with the form factor $\mu$, while the machining time $T_{1}$ increases with the form-factor $\mu$. Higher the slope $s$ is, higher the time $T_{1}$ is.

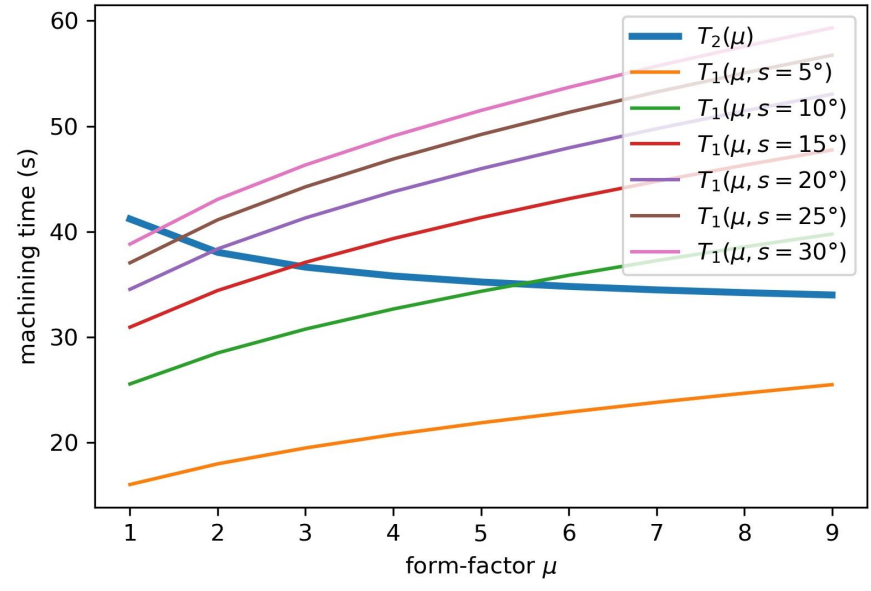

Figure 4: Evolution of machining times with respect to the form factor $\mu$ for different values of slope $s$

\subsubsection{Analysis of the analytical approximation of the machining time}

To perform a more in-depth analysis of the reliability of the hint given by the critical parameter procedure, the error in machining-time analytical calculations $\left(T_{1}\right.$ and $T_{2}$ ) should be analysed first. To do so, several simulations have been carried out, for various pairs of $s$ and $\mu$ values, constituting a grid such that $s \in\left[0^{\circ}, 90^{\circ}\right]$ and $\mu \in[1,10]$. For each pair of values, both analytical $\left(T_{1}\right.$ and $T_{2}$ ) and numerical ( $T_{1}^{*}$ and $T_{2}^{*}$, computed by machining simulation) machining times of an inclined plane are evaluated.

The relative error:

$$
\epsilon_{i}=\frac{\left|T_{i}^{*}-T_{i}\right|}{\max \left(T_{i}^{*}, T_{i}\right)} \quad \text { for } i=1,2
$$

is plotted in Figure 5. The mean relative error for machining time $T_{1}$ is $\overline{\epsilon_{1}}=0.36 \%$, while for $T_{2}$ it is $\overline{\epsilon_{2}}=4.75 \%$. As expected, the error $\epsilon_{2}$ does not depend on the slope $s$, since machining a flat surface with a spherical tool is not affected by the surface tilt angle. This error is mainly due to the approximation of the number of paths by the width (or height, according to machining direction) divided by the step-over distance, which is not an integer number. Moreover, the numerical machining simulation process uses small margins from the initial and final borders to define first and last paths. As a result, the analytic machining time overestimates its numeric counterpart by nearly the time corresponding to one path. This would also explain the fact that the error $\epsilon_{2}$ is higher than $\epsilon_{1}$ since the length of a single path is larger in the second case. Finally the analytical calculation of machining times $T_{1}$ and $T_{2}$ is considered precise enough to give a good estimation of the real machining time for both tools and directions. 


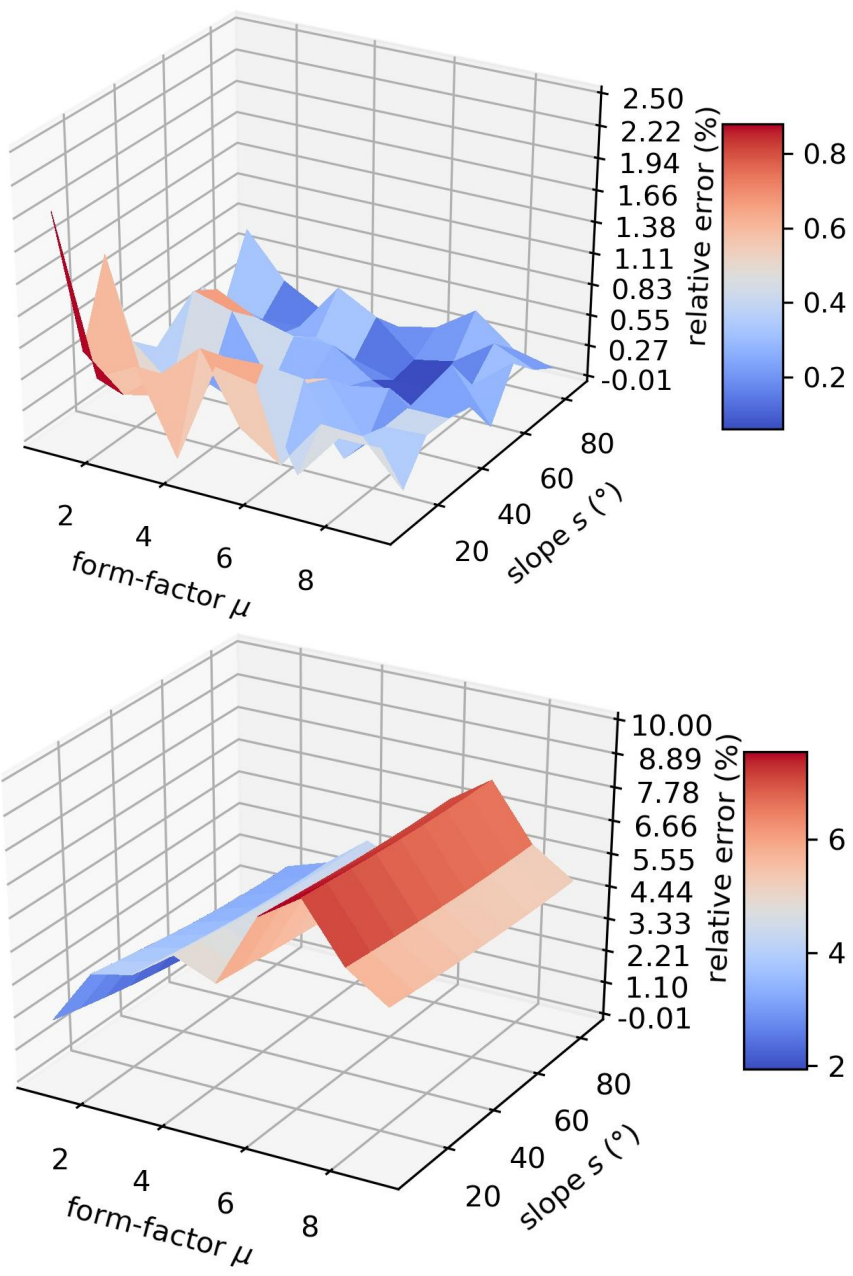

Figure 5: Machining-time relative errors: Time $T_{1}$ along the steepest-slope direction using a toroidal cutter (above), time $T_{2}$ along the principal direction using a ball-end cutter (below)

\subsubsection{Critical parameter analysis}

Based on the previous considerations, the critical parameter $s_{c}$ can be numerically analysed. Besides, in the same way that the reciprocal critical parameter $\mu_{c}$ was defined in Appendix A.1, the reciprocal analysis concerning this parameter is presented in Appendix A.2.

In order to carry out an analysis of the parameter $s_{c}$, a sample of values of the form factor $\mu$ is considered and the critical value $s_{c}$ is calculated for each $\mu$ in this sample. For each couple $\left(\mu, s_{c}(\mu)\right)$, the machining simulations are then carried out to provide the machining times $T_{1}^{*}$ and $T_{2}^{*}$. Considering this methodology, $T_{1}^{*}$ and $T_{2}^{*}$ should then be equal for each couple $\left(\mu, s_{c}(\mu)\right)$. Figure 6 shows the evolution of $s_{c}(\mu)$ and the relative error of the difference between the times $T_{1}^{*}(\mu)$ and $T_{2}^{*}(\mu)$.

The error is around $5 \%$, which confirms the validity of the critical-parameter expressions. It is worth noting that the error is higher for larger form factors. Indeed,
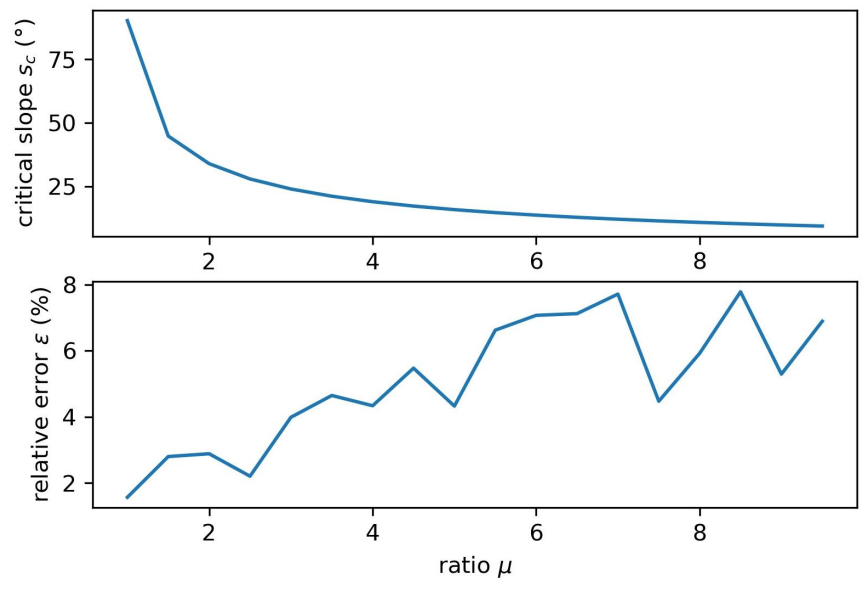

Figure 6: Evolution of the critical slope $s_{c}(\mu)$ and the machining-time relative error $\epsilon_{c}(\mu)$ with respect to the form factor $\mu$

in this case the paths along the principal direction are longer, and the approximation on the paths number has a greater impact.

To sum up, the machining times analytically calculated and the critical parameters were numerically checked. Hence, the proposed approach allows one to select, for a given plane surface, the most efficient tool geometry and machining direction without calculating machining times, but only using the slope and form factor, which is straightforward for rectangular, flat surfaces. To do so, given a plane, its slope $s$ and its form factor $\mu$, the slope $s$ can be compared to the critical slope $s_{c}(\mu)$. Then, the toroidal cutter should be privileged if $s<s_{c}(\mu)$. Otherwise, the ball-end cutter should be selected.

\section{$5 \quad$ Extension to free-form surfaces}

The objective of this section is to extend the proposed approach on flat, rectangular surfaces to general free-form surfaces. First, the theoretical framework is presented, aiming to give a definition of the critical parameters. On the base of these critical parameters, an efficient selection of cutter type and machining direction can be performed. Then, the formulated approach is benchmarked with two free-form surface test cases, in order to investigate its validity.

\subsection{Preliminary considerations}

The first question that arises when generalizing to freeform surfaces is how to define the machining directions. To do so, a $(u, v)$ mesh defined by isoparametric curves is considered in order to obtain a tessellation of the surface. This way, a bunch of elementary surface quads is obtained. Then, for each quad, a sample point is defined as the center of the quad. The machining direc- 
tions are defined using these sample points denoted $\mathbf{S}_{\mathbf{i}}$, for $i \in\{0, \ldots, n\}$.

In the case of a plane, the steepest-slope direction is the same whatever the point of the surface taken in consideration. For a free-form surface, the steepest-slope direction may vary for any point taken into consideration. As a first approach, the steepest-slope direction of a free-form surface is estimated by the average of the steepest-slope directions calculated at each sample point.

For a rectangular, flat surface, the principal direction, along which the surface is the most extended, was parallel to its largest border. It could be seen also, for any surface, as the direction of the line that minimizes the sum of squared distances from the points of the surface to their orthogonal projection on that line. According to the tessellation of the surface previously defined, this sum can be calculated discretely, relying on the sample points $\mathbf{S}_{\mathbf{i}}$.

According to these considerations, the principal direction corresponds to the line that minimizes the sum of the squared distances $d_{i}^{2}$ from the sample points $\mathbf{S}_{\mathbf{i}}$ to their orthogonal projection on that line. This principle is illustrated on Figure 7 for a few sample points. Thus, finding the principal direction is equivalent to the wellknown Principal Component Analysis (PCA) method [24] that provides the "best fit" for a data cloud.

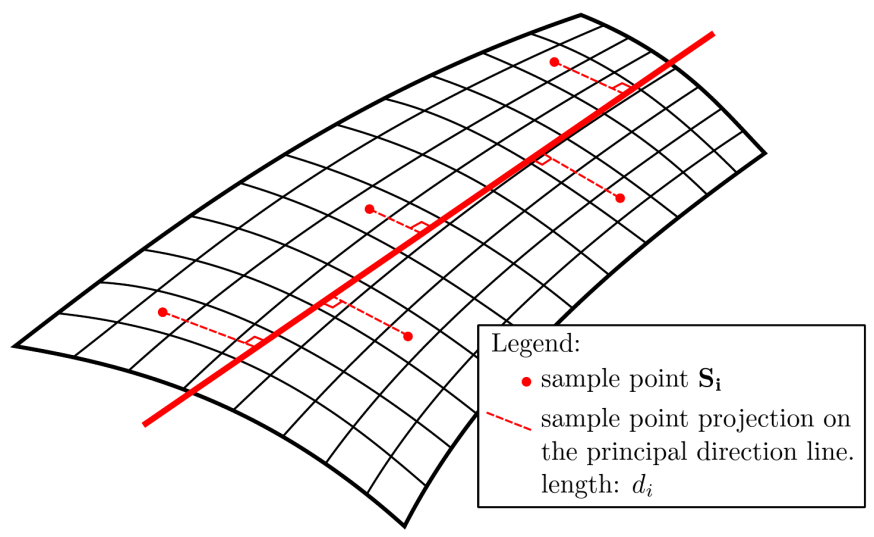

Figure 7: Evaluation of the principal direction for a freeform surface: Principle

\subsection{Using the covariance matrix to define the principal direction}

Principal Component Analysis is a multivariate statistical procedure for data analysis. It is mainly used for dimension reduction: It aims to condense the information contained in a large number of original variables into a smaller set of new orthogonal variables by means of linear combinations, with a minimum of information loss. PCA is also used for interpretation of large data sets, it can reveals relationships between correlated variables. Mathematically, PCA depends upon the eigen decomposition of positive semi-definite matrices, and upon the Singular Value Decomposition (SVD) of rectangular matrices.

In our context, for a given surface $\mathcal{S}$, the variables are the coordinates of surface points in the three-di-mensional Euclidean space: $\left(S_{x}, S_{y}, S_{z}\right)$. PCA is performed over the sample points $\mathbf{S}_{\mathbf{i}}$ through eigen decomposition of its covariance matrix, which gives three orthogonal eigenvectors $\mathbf{S}_{\mathbf{I}}, \mathbf{S}_{\mathbf{I I}}$ and $\mathbf{S}_{\mathbf{I I I}}$ corresponding to the eigenvalues $\lambda_{I} \geq \lambda_{I I} \geq \lambda_{I I I}$. Note that $\mathbf{S}_{\mathbf{I}}$ is the vector that fits the best the sample points $\mathbf{S}_{\mathbf{i}}$. Indeed, it minimizes the sum of the squared distances from the sample points to the orthogonal projection on itself. Thus, $\mathbf{S}_{\mathbf{I}}$ is considered the principal direction of the free-form surface $\mathcal{S}$. The covariance matrix $C$ of the sample points $\mathbf{S}_{\mathbf{i}}$ is

$$
C=\left(\begin{array}{ccc}
\operatorname{Var}\left(S_{x}\right) & \operatorname{Cov}\left(S_{x} S_{y}\right) & \operatorname{Cov}\left(S_{x} S_{z}\right) \\
\operatorname{Cov}\left(S_{x} S_{y}\right) & \operatorname{Var}\left(S_{y}\right) & \operatorname{Cov}\left(S_{y} S_{z}\right) \\
\operatorname{Cov}\left(S_{x} S_{z}\right) & \operatorname{Cov}\left(S_{y} S_{z}\right) & \operatorname{Var}\left(S_{z}\right)
\end{array}\right),
$$

where $\operatorname{Var}\left(S_{x}\right)=\mathrm{E}\left(S_{x}^{2}\right)-\mathrm{E}\left(S_{x}\right)^{2}$

$$
\begin{aligned}
& \operatorname{Var}\left(S_{y}\right)=\mathrm{E}\left(S_{y}^{2}\right)-\mathrm{E}\left(S_{y}\right)^{2} \\
& \operatorname{Var}\left(S_{z}\right)=\mathrm{E}\left(S_{z}^{2}\right)-\mathrm{E}\left(S_{z}\right)^{2}
\end{aligned}
$$

and $\operatorname{Cov}\left(S_{x} S_{y}\right)=\mathrm{E}\left(S_{x} S_{y}\right)-\mathrm{E}\left(S_{x}\right) \mathrm{E}\left(S_{y}\right)$

$\operatorname{Cov}\left(S_{x} S_{z}\right)=\mathrm{E}\left(S_{x} S_{z}\right)-\mathrm{E}\left(S_{x}\right) \mathrm{E}\left(S_{z}\right)$

$\operatorname{Cov}\left(S_{y} S_{z}\right)=\mathrm{E}\left(S_{x} S_{z}\right)-\mathrm{E}\left(S_{x}\right) \mathrm{E}\left(S_{z}\right)$.

In these expressions, $\mathrm{E}(X)$ is the average of quantity $X$ over the $n$ sample points previously defined. Eigenvectors are orthogonal because the covariance matrix is symmetric. Since the cutter must remain tangent to surface during machining, and surfaces are geometric varieties of dimension 2, the eigenvector $\mathbf{S}_{\mathbf{I I}}$ represents the less-extended surface direction, which explain that best results will be obtained when the steepest-slope direction is near the perpendicular to the principal direction $\mathbf{S}_{\mathbf{I}}$ (More considerations on this point are discussed in Section 7.1). The eigenvector $\mathbf{S}_{\text {III }}$ is not considered since $\lambda_{I I I}$ is equal to zero for flat surfaces. In general, the smaller $\lambda_{I I I}$ is, the flater the surface is.

\subsection{Using eigenvalues to define an ap- proximate form factor of a free-from surface}

It is worth noting that the eigenvectors $\mathbf{S}_{\mathbf{I}}$ and $\mathbf{S}_{\mathbf{I I}}$ span the plane that fits best the surface $\mathcal{S}$ (this should be understood in terms of minimizing the sum of squared distances from sample points $\mathbf{S}_{\mathbf{i}}$ to their orthogonal projection on this plane). The eigenvalues $\lambda_{I}$ and $\lambda_{I I}$ represent the variance of the projected points on $\mathbf{S}_{\mathbf{I}}$ and $\mathbf{S}_{\mathbf{I I}}$, respectively. It is straightforward, that the projection of a continuous surface on a vector is a continuous segment whose length is proportional to the standard deviation of the projected points. As a result, the width/height ratio of a free-form surface can be approximated by the square root of the eigenvalues fraction and the characteristic size by the square root of the eigenvalues product. 
Consequently, an approximated form factor $\tilde{\mu}$ of a freefrom surface can be defined as:

$$
\tilde{\mu}=\sqrt{\frac{\lambda_{I}}{\lambda_{I I}}} .
$$

In the same way, an approximated characteristic size $\tilde{\chi}$ of a free-from surface may be defined:

$$
\tilde{\chi}=12 \sqrt{\lambda_{I} \lambda_{I I}}
$$

The word "approximated" must be understood in the sense that these definitions depend on the mesh size. However, it has been empirically observed that the mesh size does not significantly change these values as long as it is chosen sufficiently large. Moreover, it has been proven (see Appendix B) that when continuously and uniformly sampling the flat, rectangular surfaces $\tilde{\mu}$ (respectively $\tilde{\chi}$ ) coincides with $\mu=\frac{w}{h}$ (respectively with $\chi=w h$ ).

\subsection{Approximated slope of a free-form surface}

Contrary to flat surfaces, the steepest-slope of free-form surfaces is not constant. As proposed in Section 5.1, a first approximation could be the mean of sample points slopes over the entire surface. In this case, the approximating slope is given by:

$$
\tilde{s}=\mathrm{E}\left(s_{i}\right)
$$

where $s_{i}$ is the slope of the sampling point $\mathbf{S}_{\mathbf{i}}$.

Another approximation, relying on PCA, consists in using the slope of the plane spanned by the eigenvectors $\mathbf{S}_{\mathbf{I}}$ and $\mathbf{S}_{\mathbf{I I}}$, since it is the plane that best fits the surface:

$$
\tilde{s}=\frac{\pi}{2}-\arccos \left(\frac{\mathbf{S}_{\mathbf{I}} \times \mathbf{S}_{\mathbf{I I}}}{\left\|\mathbf{S}_{\mathbf{I}} \times \mathbf{S}_{\mathbf{I I}}\right\|} \cdot \mathbf{Z}\right)
$$

which gives the expression of the approximated slope as a function of eigenvectors. Both approximations are exact for flat surfaces.

In other words, the steepest-slope of a free-form surface $\mathcal{S}$ may be approximated either by the mean of steepestslopes over the surface, or by the steepest-slope of the plane spanned by $\mathbf{S}_{\mathbf{I}}$ and $\mathbf{S}_{\mathbf{I I}}$. The influence of this choice is discussed in Section 7.3, but from a purely computational point of view the second method avoids the calculation of the slope at each sample point (and therefore the calculation of the normal vector $\mathbf{n}_{\mathbf{i}}$ at each sample point $\mathbf{S}_{\mathbf{i}}$ ).

In the same way, the steepest-slope direction may be calculated either as the mean of all the steepest-slope directions calculated at each sample point $\mathbf{S}_{\mathbf{i}}$ or as the projection of $\mathbf{S}_{\mathbf{I}} \times \mathbf{S}_{\mathbf{I I}}$ on the horizontal plane $(\mathbf{X}, \mathbf{Y})$.

\section{Practical application to free- form surfaces}

\subsection{Step-by-step description of the method to choose the best cutter type}

This section is devoted to the practical application of the method we propose based on previously demonstrated results. Remark that no machining simulation is needed to perform this method. In order to set up such a method few data are needed. Obviously, the machine characteristics $A_{\max }$ and $J_{\max }$, and the milling operation data $V$, $R, r$ and $s h$ are required. Furthermore, a set of sample points from the surface to be machined is also needed. These points can be extracted for a CAD representation of the surface, measured on a prototype part, or obtained by any other method.

1. Define a set of sample points $\mathbf{S}_{\mathbf{i}}$, over the entire surface $\mathcal{S}$. Only the $\left(S_{x}, S_{y}, S_{z}\right)$ coordinates of these points are needed for the calculations that follows.

2. Calculate the covariance matrix $C$ using equation (16)

3. Perform eigen decomposition of $C$ to find $\lambda_{I}, \lambda_{I I}$, $\mathbf{S}_{I}, \mathbf{S}_{\text {II }}$

4. Calculate the steepest-slope $\tilde{s}$ using Equation (20) and the steepest-slope direction

5. Calculate the form factor $\tilde{\mu}$ and the characteristic size $\tilde{\chi}$ using Equations (17) and (18)

6. Calculate the critical parameter $s_{c}(\tilde{\mu})$ using Equation (14)

7. Compare $\tilde{s}$ with the critical parameter: if $s_{c}(\tilde{\mu})<\tilde{s}$, then choose the ball-end cutter along the principal direction. Otherwise, select the toroidal cutter along the steepest-slope direction.

Remark that most stages of this procedure are direct applications of analytical formulas. This means that these stages are really quick to perform. The main piece of computation is the eigen decomposition of the covariance matrix $C$. Several sofware packages are very efficient to perform this task, especially on small $(3 \times 3)$ matrices.

\subsection{Test cases}

In order to test the aforementioned approach, a benchmark with two test-case free-form surfaces is designed. Both surfaces are chosen so that the principal direction is nearly orthogonal with the approximation of the steepestslope direction, and discretised using a regular isoparametric $80 \times 80$ mesh. The machining times, corresponding to toroidal cutter along steepest-slope direction, and 
ball-end cutter along principal direction, are compared and used to confirm the result predicted by the critical parameters $\mu_{c}(\tilde{s})$ and $s_{c}(\tilde{\mu})$.

\subsubsection{Test case 1}

The first test case is a Bézier surface of $3 \times 3$ control points given in Table 2 .

\begin{tabular}{|c|c|c|}
\hline point $(0,0)$ & point $(0,1)$ & point $(0,2)$ \\
\hline 0.0 & 0.0 & 0.0 \\
-15.32 & -6.42 & 15.32 \\
-12.85 & 7.66 & 12.85 \\
\hline point $(1,0)$ & point $(1,1)$ & point $(1,2)$ \\
\hline 40.0 & 40.0 & 40.0 \\
-18.53 & -9.64 & 12.10 \\
-9.02 & 11.49 & 16.68 \\
\hline point $(2,0)$ & point $(2,1)$ & point $(2,2)$ \\
\hline 80.0 & 80.0 & 80.0 \\
-28.17 & -22.49 & 2.46 \\
2.46 & 26.81 & 28.17 \\
\hline
\end{tabular}

Table 2: Test surface 1: cartesian coordinates of control points

This surface have been used in $[9,25]$ and it is illustrated in Figure 8 where the axis $\mathbf{X}, \mathbf{Y}$ and $\mathbf{Z}$ respectively, is represented by the red, green and blue vector, respectively.

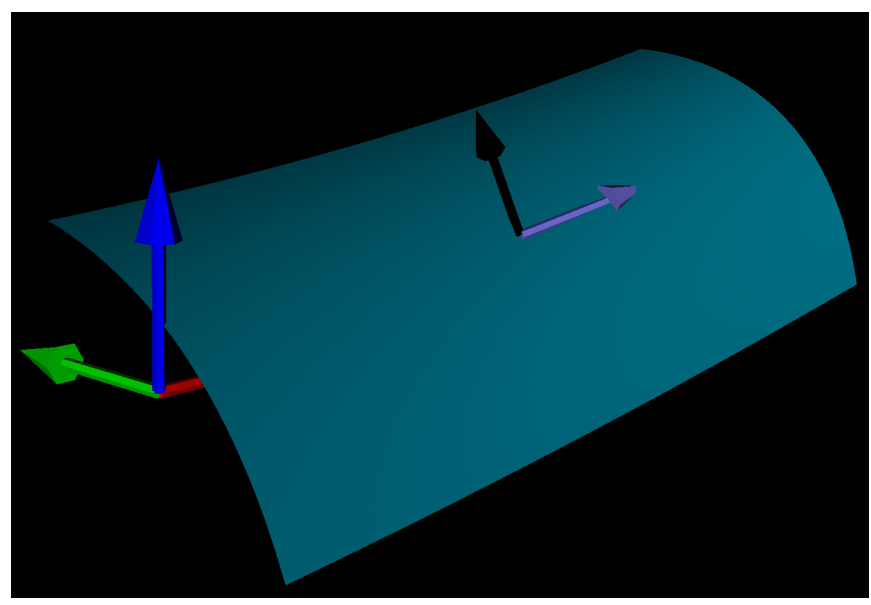

Figure 8: Test surface 1 with its principal (purple) and steepest-slope (black) directions

On this figure, the black vector indicates the steepestslope direction while the purple arrow shows the principal direction. The approximated slope $\tilde{s}$, calculated using Equation (20), is equal to $0.739 \mathrm{rad}=42.34^{\circ}$. The steepest-slope direction (speepest slope vector projected onto the ( $\mathbf{X}, \mathbf{Y})$ plane) forms an angle with axis $\mathbf{X}$ equal to $1.169 \mathrm{rad}=66.97^{\circ}$. The PCA performed on the surface enables the calculation of the eigenvalues: $\lambda_{I}=572.62$

\begin{tabular}{|c|c|c|}
\hline & machining time & toolpath length \\
\hline $\begin{array}{c}\text { toroidal tool } \\
\text { along steepest- } \\
\text { slope direction }\end{array}$ & $91.3 \mathrm{~s}$ & $5606 \mathrm{~mm}$ \\
\hline $\begin{array}{c}\text { ball-end tool } \\
\text { along principal } \\
\text { direction }\end{array}$ & $79.7 \mathrm{~s}$ & $5687 \mathrm{~mm}$ \\
\hline
\end{tabular}

Table 3: Machining times and toolpath lengths for test surface 1

and $\lambda_{I I}=133.31$, which yield the form factor: $\tilde{\mu}=2.07$, and the principal direction, defining an angle of $-0.17 \mathrm{rad}$ $=-9.74^{\circ}$ with the axis $\mathbf{X}$. Results of machining along both directions are summarized in Table 3. The numerical simulation of machining takes nearly $3 \mathrm{~s}$. Figure 9 displays the machining toolpaths in both cases.

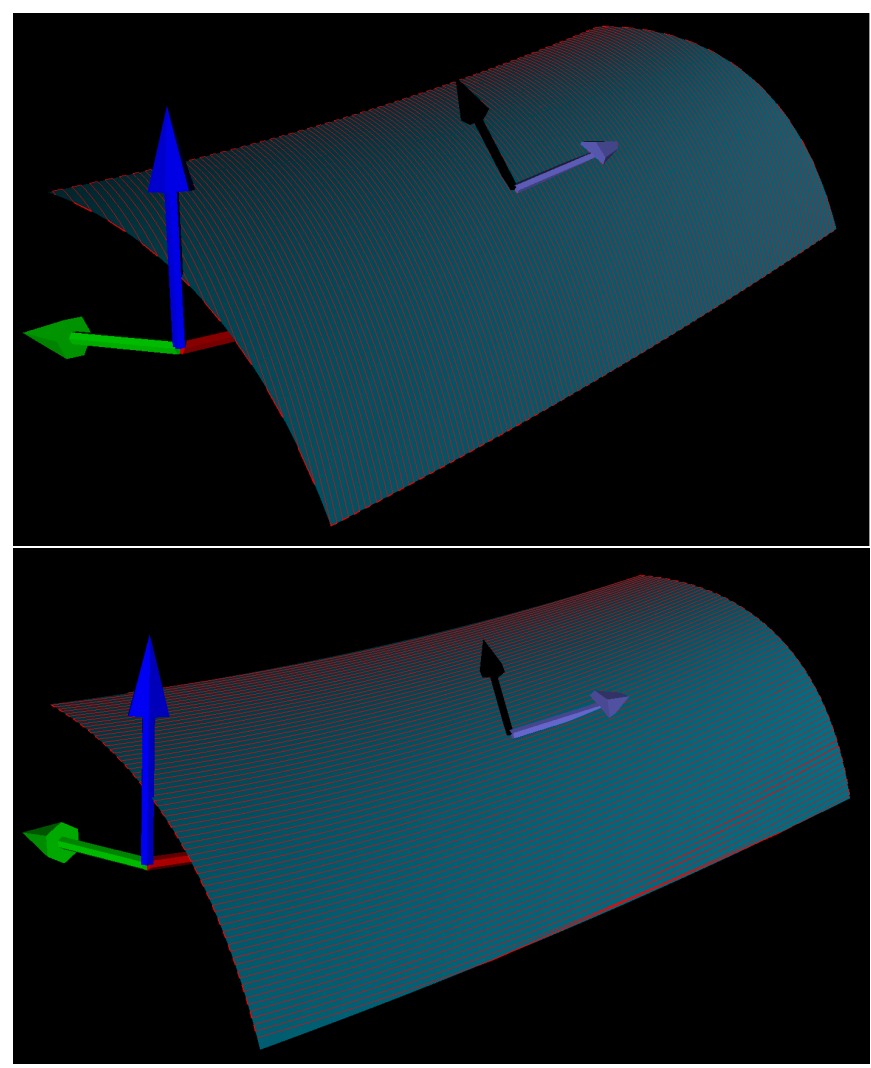

Figure 9: Machining of test case surface 1: along the steepest-slope direction using a toroidal cutter (above), along the principal direction using a ball-end cutter (below)

From the obtained results, one remarks the importance of taking account the kinematics of the CNC machine. In fact, eventhough the toolpath length is slightely shorter for the toroidal tool, the ball-end tool features a shorter machining time.

Testing the proposed procedure on this surface is conducted as follows: The characteristic size $\tilde{\chi}$ of the plane 
spanned by eigenvectors is deduced from the eigenvalues:

$$
\tilde{\chi}=12 \sqrt{\lambda_{I} \lambda_{I I}}=3315.47 \mathrm{~mm}^{2}
$$

Knowing that $\tilde{\mu}=2.07$, the critical slope can be calculated using Equation (14): $s_{c}(\tilde{\mu})=38.9^{\circ}$. Since $s_{c}<\tilde{s}$, our approach predicts that the ball-end tool will present a shorter machining time, which agrees with the numerical results. Consequently, the prediction is correct on this first test case.

\subsubsection{Test case 2}

The second test case is a Bézier surface of $4 \times 4$ control points given in Table 4 . This surface was previ-

\begin{tabular}{|c|c|c|c|}
\hline point $(0,0)$ & point $(0,1)$ & point $(0,2)$ & point $(0,3)$ \\
\hline 7.92 & 6.33 & 6.33 & 7.92 \\
0.0 & 20.4 & 40.8 & 61.2 \\
37.26 & 29.81 & 29.81 & 37.26 \\
\hline point $(1,0)$ & point $(1,1)$ & point $(1,2)$ & point $(1,3)$ \\
\hline 23.72 & 22.14 & 22.14 & 23.72 \\
0.0 & 20.4 & 40.8 & 61.2 \\
26.11 & 18.66 & 18.66 & 26.11 \\
\hline point $(2,0)$ & point $(2,1)$ & point $(2,2)$ & point $(2,3)$ \\
\hline 42.70 & 41.12 & 41.12 & 42.70 \\
0.0 & 20.4 & 40.8 & 61.2 \\
29.87 & 22.42 & 22.42 & 29.87 \\
\hline point $(3,0)$ & point $(3,1)$ & point $(3,2)$ & point $(3,3)$ \\
\hline 56.02 & 54.44 & 54.44 & 56.02 \\
0.0 & 20.4 & 40.8 & 61.2 \\
19.25 & 11.79 & 11.79 & 19.25 \\
\hline
\end{tabular}

Table 4: Test surface 2: cartesian coordinates of control points

ously used in [26] for free-form surface machining tests. It is illustrated in Figure 10, with the same color code as for the previous test case for axes $\mathbf{X}, \mathbf{Y}$ and $\mathbf{Z}$. Again, the steepest-slope and the principal directions are nearly orthogonal. The approximated slope $\tilde{s}$ is equal to $0.299 \mathrm{rad}=17.13^{\circ}$, and the steepest-slope direction is aligned with axis $\mathbf{X}$. The eigenvalues are $\lambda_{I}=312.07$ and $\lambda_{I I}=219.02$, which are used to calculate the form factor: $\tilde{\mu}=1.19$, and the principal direction, defining an angle of $1.57 \mathrm{rad}=90.0^{\circ}$ with the axis $\mathbf{X}$. The results of machining along both directions are summarized in Table 5. The numerical simulation of machining takes nearly $3 \mathrm{~s}$. Figure 11 displays the machining toolpaths in both cases. Here the toolpath length is slightely shorter for the toroidal tool, and this cutter is also the one presenting the shorter machining time.

Testing the proposed procedure on this surface is conducted as follows: The characteristic size of the plane spanned by eigenvectors is equal to $\tilde{\chi}=3137 \mathrm{~mm}^{2}$; note that this value is close to the area of the surface.

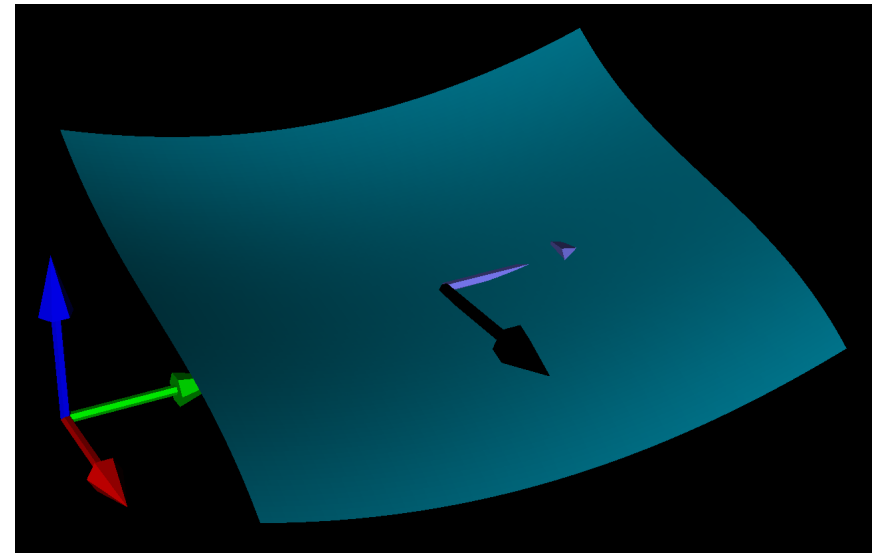

Figure 10: Test surface 2 with its principal (purple) and steepest-slope (black) directions

\begin{tabular}{|c|c|c|}
\hline & machining time & toolpath length \\
\hline $\begin{array}{c}\text { toroidal tool } \\
\text { along steepest- } \\
\text { slope direction }\end{array}$ & $71.8 \mathrm{~s}$ & $4725 \mathrm{~mm}$ \\
\hline $\begin{array}{c}\text { ball-end tool } \\
\text { along principal } \\
\text { direction }\end{array}$ & $76.8 \mathrm{~s}$ & $5184 \mathrm{~mm}$ \\
\hline
\end{tabular}

Table 5: Machining times and toolpath lengths for test surface 2

Knowing that $\tilde{\mu}=1.19$, the critical slope can be calculated using Equation (14) and is equal to $s_{c}(\tilde{\mu})=62.6^{\circ}$. Since $\tilde{s}<s_{c}$, our approach predicts that the toroidal cutter will present a shorter machining time, which agrees with the numerical results. Consequently, the prediction is also correct on this second test case.

To investigate further the reliability of the proposed method, some tests are carried out to determinate numerically the critical slope $s_{c}^{*}$. To do this, both machining times $T_{1}^{*}$ and $T_{2}^{*}$ are estimated by numerical simulation for various values of the surface slope $\tilde{s}$. Conducting these experiments reveals equal machining times for $s_{c}^{*}=54,7^{\circ}$. Compared to the value $s_{c}=62.6^{\circ}$ provided by the proposed method, the prediction presents an error of around $10 \%$. This value should be considered cautiously, because it is heavily dependent on the surface geometry itself.

\section{Discussion}

The validity and limitations of the proposed approach are discussed in this section. 

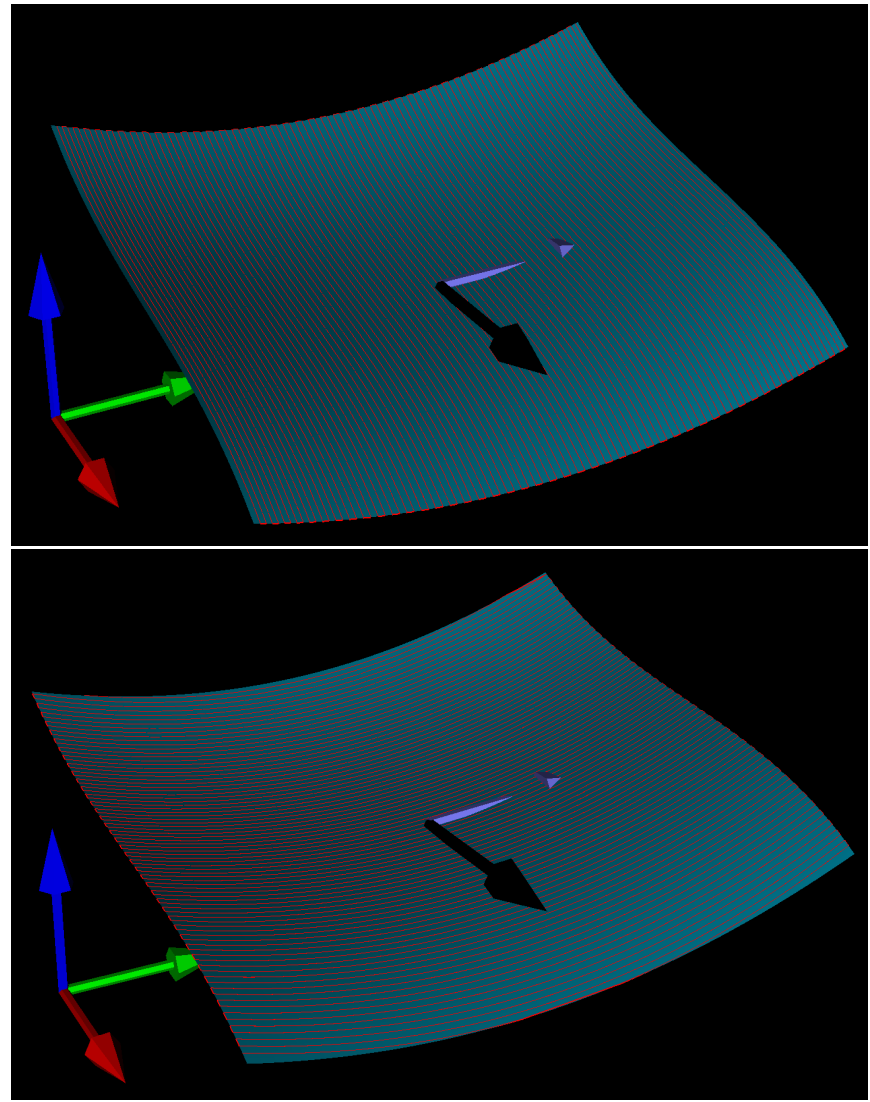

Figure 11: Machining of test case surface 2: along the steepest-slope direction using a toroidal cutter (above), along the principal direction using a ball-end cutter (below)

\subsection{On the efficiency of the proposed method}

The proposed procedure is fully efficient when machining directions (i.e., the steepest-slope direction and the surface most extended direction, called principal direction) are close to the perpendicular, otherwise the calculation of critical parameters looses its accuracy. This can be illustrated by the experiments conducted on the first test case surface (Section 6.2.1) in order to determine numerically the critical slope $s_{c}^{*}$, as it has been done for the second test case (Section 6.2.2). Performing these tests requires rotating the surface to define various values of the slope $\tilde{s}$, but rotating the surface leads to significant change of the steepest-slope direction, which is no longer orthogonal to the principal direction. Therefore, the prediction is correct in the range of slopes $\tilde{s}$ such that both directions are nearly orthogonal, but it becomes quickly erroneous when both directions are too far from perpendicularity. Thus, the closer the machining directions are to the perpendicular, the more accurate is the calculation of the critical parameters. This may appear like quite a limiting factor, but actually the closer the steepest-slope and the principal directions are, the more the toroidal tool should be privileged, since it leads to larger step-over distances without increasing the number of paths. Therefore, the proposed procedure is really useful and accurate for really problematic surfaces, and less interesting for surfaces for which machining directions and best cutter are obvious.

\subsection{Limitations of scope}

The proposed approach is analytically proved for flat rectangular surfaces. To do so a few hypotheses were made:

- First, the $\frac{\chi}{\mu}$ ratio is considered great enough to ensure that path machining time is calculated such that nominal feed rate is achieved before the maximum acceleration. For the kinematic parameters values used in this paper, the lower bound is $\frac{\chi}{\mu}>7.6 \mathrm{~mm}$. This condition excludes very small surfaces, which is acceptable in practice.

- Secondly, it is supposed that step-over distances (between two adjacent paths) are not sufficiently long to achieve nominal feed rate. This is verified for both cutters as soon as the machining strip width is inferior to $7.6 \mathrm{~mm}$. This condition depends on the kinematic parameter values but also on the cutter dimensions, and needs to be verified when these values change. In practice, the size of cutters used for finishing are small enough to consider this condition verified.

- The last hypothesis concerns the calculation of the critical slope $s_{c}$. It was supposed that $\chi>100 \mathrm{~mm}^{2}$ in order to ensure that Equation (10) has only one real solution. In general, the cases where the surface area is smaller than $100 \mathrm{~mm}^{2}$ are very rare.

Furthermore, the proposed approach relies strongly on the kinematic parameters of the NC machine-tool: $J_{\max }$, $A_{\max }$ and the nominal feed rate $V$. Therefore, the hypotheses concerning the machining time calculation in Section 2.2 need to be checked for different values of kinematic parameters, but the procedure remains the same.

\subsection{On the extension to free-form sur- faces}

The proposed approach is then extended to free-form surfaces. Actually, the approach is more accurate for surfaces with slowly-varying normal vectors, and thereby slowly-varying steepest-slope directions. On the other side, the approach is less accurate when the steepestslope direction varies much, especially when the parallelplanes strategy is used. Indeed, the parallel-planes strategy implies that the step-over distance is determined by the worst interpolation point, contrary to the iso-scallop 
strategy, and the range of calculated step-over distances is larger when the surface normals vary much.

For free-form surfaces, there are two ways, as mentioned before (Section 5.4), to calculate the approximated slope $\tilde{s}$ that might give different values. Again, the difference here is larger when the normal vector varies much. However, through all tests completed while working on this issue, choosing one method over the other never lead to a difference significant enough to make an erroneous prediction.

Remark that if the user is compelled to only use the ball-end cutter, the principal direction is a machining direction of choice, since it reduces the number of paths, compared to other directions at a constant step-over distance. Besides, identifying the principal direction may be interesting in 5-axis and $3+2$-axis machining with toroidal cutter. Indeed, the two rotary angles can be set up so that the steepest-slope and principal directions coincides, which will improve both the step-over distances and the number of paths, reducing considerably the machining time.

Another point that is worth to be noted is that the scallop height tolerance $s h$ does not seem to have a noteworthy influence on the critical parameters. Indeed, the same evolution curve of critical parameter $s_{c}(\mu)$ (see Figure 6 ) is found for $s h=0.1 \mathrm{~mm}$.

The impact of the mesh size is also investigated because both machining directions defined previously (steepestslope and principal) are calculated from the sampled points $\mathbf{S}_{\mathbf{i}}$ on the mesh, and thus depend on the mesh size. Numerous tests for different meshing sizes (from $20 \times 20$ to $200 \times 200$ ) had been run, and it was found that the change on machining times is not significant, especially for mesh sizes greater than $60 \times 60$. The parameters $\tilde{s}$ and $\tilde{\mu}$ depend also on the mesh size, and similar observations were found. Hence, the mesh size can be chosen big enough to ensures that results are not sensitive to it.

\subsection{Influence of auxiliary parameters}

All along this study, the characteristic size $\chi$ is considered constant. Indeed, this parameter, representing the area of the surface, has much less influence than the other parameter, $\mu$, representing the elongation of the surface. Nevertheless, its effect is examined here, despite its weak role. Machining along the principal direction using a ballend cutter is more efficient compared to its counterpart (torus-end mill along greatest slope direction), when the paths generated in the first case are such that the feed rate is achieved on a significantly larger proportion of the paths, compared to the second case. Thus, for a given feed rate $V$, the more the surface area is large, the bigger is the proportion of paths for which the feed rate is achieved for both machining cases, promoting the toroidal cutter along the steepest-slope direction. As a result, the critical parameter $s_{c}$ is expected to increase as $\chi$ increases. This statement is proven in Appendix D.

Similarly, the feed rate $V$ was considered fixed. Yet, it can be shown that a high feed rate promotes machining along the principal direction, while a low feed rate favours the slope direction. More precisely, the feed rate is achieved on larger portions of toolpath as its value is smaller, and the interest of the principal direction is lost in that case. Conversely, a high feed rate would penalize toolpaths with more direction changes, thus promoting machining along the principal direction. This statement is confirmed by numerical simulations showing that the critical parameters $\mu_{c}$ and $s_{c}$ are decreasing functions of $V$.

The proposed method performs PCA over the surfacepoint coordinates in order to find the principal direction and estimate parameters $\tilde{\mu}$ and $\tilde{\chi}$ using eigenvalues $\lambda_{I}$, $\lambda_{I I}$ and their eigenvectors. Although the third eigenvector $\lambda_{I I I}$ is not used in our method, it can give a global idea about the flatness of a given surface and thus the applicability of the proposed method to this surface. Quantitative criteria are not available at this stage, but some local parameters, such as the Gaussian curvature, should be taken into account to built them up.

\section{Conclusion and perspectives}

A new method of selection between the ball-end mill and the toroidal cutter is presented in this article. The most important advantage of this method is that it provides a reliable information, without performing a machining simulation. The method is first tested for flat surfaces, then extended to free-form surfaces using the analysis of main components. It is based on the identification of two characteristic machining directions: the steepest-slope direction and the principal direction, the first favoring the toroidal tool while the second favors the spherical tool. The approach has given the best results when these two directions are antagonistic (i.e., perpendicular), but when they are not, the choice of the type of mill is generally obvious. The proposed method also works best when the normal vector at the surface varies slowly, but can still provide a good clue for more complicated free-form surfaces.

This work is only a first approach to the cutter type selection problem using principal component analysis. Further works may include more accurate theoretical evaluation of the number of paths included into a toolpath, and better free-form surface parameter approximations. These future tracks of research may also be related to machining strategy, and include a full analysis of the nominal feed speed influence on the critical parameters. The third eigenvalue (equal to zero for flat surfaces) can be used to get an idea of the flatness of freeform surfaces, and therefore of the precision of the approach. This also could be the subject of new investigations. 


\section{References}

[1] Redonnet JM, Djebali S, Segonds S, Senatore J, Rubio W (2013) Study of the effective cutter radius for end milling of free-form surfaces using a torus milling cutter. Computer-Aided Design 45(6):951-962

[2] Blackmore D, Leu M (1992) Analysis of swept volume via lie groups and differential equations. The International Journal of Robotics Research 11(6):516537

[3] Chiou CJ, Lee YS (2002) A machining potential field approach to tool path generation for multi-axis sculptured surface machining. Computer-Aided Design $34(5): 357-371$

[4] Kumazawa GH, Feng HY, Barakchi Fard MJ (2015) Preferred feed direction field: A new tool path generation method for efficient sculptured surface machining. Computer-Aided Design 67-68:1-12

[5] Liu X, Li Y, Ma S, Lee Ch (2015) A tool path generation method for freeform surface machining by introducing the tensor property of machining strip width. Computer-Aided Design 66:1-13

[6] Mann S, Bedi S (2002) Generalization of the imprint method to general surfaces of revolution for NC machining. Computer-Aided Design 34(5):373-378

[7] Roth D, Bedi S, Ismail F, Mann S (2001) Surface swept by a toroidal cutter during 5 -axis machining. Computer-Aided Design 33(1):57-63

[8] Sheltami K, Bedi S, Ismail F (1998) Swept volumes of toroidal cutters using generating curves. International Journal of Machine Tools and Manufacture $38(7): 855-870$

[9] Vu DD, Monies F, Segonds S, Rubio W (2020) Automatic minimal partitioning method guaranteeing machining efficiency of free-form surfaces using a toroidal tool. The International Journal of Advanced Manufacturing Technology 107:4239-4254

[10] Vickers GW, Quan KW (1989) Ball-Mills Versus End-Mills for Curved Surface Machining. Journal of Engineering for Industry 111(1):22-26

[11] Bedi DS, Ismail F, Mahjoob MJ, Chen Y (1997) Toroidal versus ball nose and flat bottom end mills. The International Journal of Advanced Manufacturing Technology 13(5):326-332

[12] Cho H, Jun Y, Yang M (1993) Five-axis CNC milling for effective machining of sculptured surfaces. International Journal of Production Research 31(11):2559-2573
[13] Kim B, Chu C (1994) Effect of cutter mark on surface roughness and scallop height in sculptured surface machining. Computer-Aided Design 26(3):179188

[14] Djebali S, Perles A, Lemouzy S, Segonds S, Rubio W, Redonnet JM (2015) Milling plan optimization with an emergent problem solving approach. Computers \& Industrial Engineering 87:506-517

[15] Djebali S, Segonds S, Redonnet JM, Rubio W (2015) Using the global optimisation methods to minimise the machining path length of the free-form surfaces in three-axis milling. International Journal of Production Research 53(17):5296-5309

[16] Griffiths JG (1994) Toolpath based on Hilbert's curve. Computer-Aided Design 26(11):839-844

[17] Lazoglu I, Manav C, Murtezaoglu Y (2009) Tool path optimization for free form surface machining. CIRP Annals 58(1):101-104

[18] Makhanov S (2007) Optimization and correction of the tool path of the five-axis milling machine: Part 1. Spatial optimization. Mathematics and Computers in Simulation 75(5):210-230

[19] Park SC (2003) Tool-path generation for Zconstant contour machining. Computer-Aided Design 35(1):27-36

[20] Park SC, Choi BK (2000) Tool-path planning for direction-parallel area milling. Computer-Aided Design 32(1):17-25

[21] Roman A, Bedi S, Ismail F (2006) Three-half and half-axis patch-by-patch NC machining of sculptured surfaces. The International Journal of Advanced Manufacturing Technology 29(5):524-531

[22] Pessoles X, Redonnet JM, Segonds S, Mousseigne M (2012) Modelling and optimising the passage of tangency discontinuities in NC linear paths. International Journal of Advanced Manufacturing Technology 58(5-8):631-642

[23] Pessoles X, Landon Y, Rubio W (2010) Kinematic modelling of a 3-axis NC machine tool in linear and circular interpolation. The International Journal of Advanced Manufacturing Technology 47(5-8, SI):639-655

[24] Abdi H, Williams LJ (2010) Principal component analysis. Wiley Interdisciplinary Reviews: Computational Statistics 2(4):433-459

[25] Vu DD, Monies F, Rubio W (2018) A new optimization tool path planning for 3 -axis end milling of freeform surfaces based on efficient machining intervals. AIP Conference Proceedings 1960(1):070011 
[26] Choi YK (2007) Tool path generation and tolerance analysis for free-form surfaces. International Journal of Machine Tools and Manufacture 47(3-4):689-696

\section{Appendices}

\section{A Study of the critical parameter

$$
\mu_{c}=f(s)
$$

The expression of the critical parameter $s_{c}=f(\mu)$ has been developed in Section 4.1.3. The reciprocal function $\mu_{c}=f(s)$ is far less interesting from a practical point of view, however some use cases may be thought up, especially at the conception stage of the part.

\section{A.1 Analytical expression of $\mu_{c}$}

For a given slope $s$, the critical form factor $\mu_{c}$ is defined such as the machining times $T_{1}$ and $T_{2}$ asociated to both cutters and directions are equal:

$$
\begin{aligned}
T_{1}= & T_{2} \\
\Leftrightarrow & \frac{\sqrt{\mu \chi}}{\operatorname{sod}_{1}}\left(\frac{\sqrt{\chi}}{\sqrt{\mu} V}+2 \sqrt{\frac{V}{J_{\max }}}+4 \sqrt[3]{\frac{\operatorname{sod}_{1}}{2 J_{\max }}}\right) \\
& =\frac{\sqrt{\chi}}{\sqrt{\mu} \operatorname{sod}_{2}}\left(\frac{\sqrt{\chi \mu}}{V}+2 \sqrt{\frac{V}{J_{\max }}}+4 \sqrt[3]{\frac{\operatorname{sod}_{2}}{2 J_{\max }}}\right) \\
\Leftrightarrow & \frac{\sqrt{\chi \mu}}{V}+2 \sqrt{\frac{V}{J_{\max }}}+4 \sqrt[3]{\frac{\operatorname{sod}_{2}}{2 J_{\max }}} \\
& =\mu \frac{\operatorname{sod}_{2}}{\operatorname{sod}_{1}}\left(2 \sqrt{\frac{V}{J_{\max }}}+4 \sqrt[3]{\frac{\operatorname{sod}_{1}}{2 J_{\max }}}\right)+\mu \frac{\operatorname{sod}_{2}}{\operatorname{sod}_{1}} \frac{\sqrt{\chi}}{\sqrt{\mu} V}
\end{aligned}
$$

Thus, $T_{1}=T_{2}$ is equivalent to

$$
\begin{aligned}
& \frac{\operatorname{sod}_{2}}{\operatorname{sod}_{1}}\left(2 \sqrt{\frac{V}{J_{\max }}}+4 \sqrt[3]{\frac{\operatorname{sod}_{1}}{2 J_{\max }}}\right) \mu \\
& \quad+\frac{\sqrt{\chi}}{V}\left(\frac{\operatorname{sod}_{2}}{\operatorname{sod}_{1}}-1\right) \sqrt{\mu}-2 \sqrt{\frac{V}{J_{\max }}}-4 \sqrt[3]{\frac{\operatorname{sod}_{2}}{2 J_{\max }}}=0
\end{aligned}
$$

The last equation is a second degree polynomial in $\sqrt{\mu}$ written:

$$
a \mu+b \sqrt{\mu}+c=0,
$$

with:

$$
\begin{array}{r}
a=\frac{\operatorname{sod}_{2}}{\operatorname{sod}_{1}}\left(2 \sqrt{\frac{V}{J_{\max }}}+4 \sqrt[3]{\frac{\operatorname{sod}_{1}}{2 J_{\max }}}\right) \\
b=-\frac{\sqrt{\chi}}{V}\left(1-\frac{\operatorname{sod}_{2}}{\operatorname{sod}_{1}}\right) \\
c=-\left(2 \sqrt{\frac{V}{J_{\max }}}+4 \sqrt[3]{\frac{\operatorname{sod}_{2}}{2 J_{\max }}}\right)
\end{array}
$$

It is trivial to verify that $a$ is positive and $c$ is negative. Moreover, since $0<s<\pi / 2$, then $0<\sin s<1$ and

$$
\frac{R-r}{\sin s}+r>R \text { thus } \operatorname{sod}_{1}>\operatorname{sod}_{2} .
$$

As a result $b<0$. The discriminant of Equation (21) is $\Delta=b^{2}-4 a c>b^{2}$ and is positive. Equation (21) has therefore two solutions, one of which is negative. Indeed:

$$
\frac{-b-\sqrt{\Delta}}{2 a}=\frac{-b-\sqrt{b^{2}-4 a c}}{2 a}
$$

is negative, since $4 a c<0$ and $2 a>0$.

Therefore, the expression of critical form factor $\mu_{c}(s)$ depends on $s$ via $\operatorname{sod}_{1}$ and is given by the other solution of Equation (21). For a given value of slope $s$ :

$$
\mu_{c}=\left(\frac{\frac{\sqrt{\chi}}{V}\left(1-\frac{\text { sod }_{2}}{\text { sod }_{1}}\right)+\sqrt{\frac{\chi}{V^{2}}\left(1-\frac{\text { sod }_{2}}{\text { sod }_{1}}\right)^{2}+16 \frac{\text { sod }_{2}}{\text { sod }_{1}}\left(\sqrt{\frac{V}{J_{\max }}}+2 \sqrt[3]{\frac{s o d_{1}}{2 J_{\max }}}\right)\left(\sqrt{\frac{V}{J_{\max }}}+2 \sqrt[3]{\frac{\text { sod }_{2}}{2 J_{\max }}}\right)}}{4 \frac{\text { sod }_{2}}{\text { sod }_{1}}\left(\sqrt{\frac{V}{J_{\max }}}+2 \sqrt[3]{\frac{s o d_{1}}{2 J_{\max }}}\right)}\right)^{2}
$$

If $\mu>\mu_{c}$ then $a \mu+b \sqrt{\mu}+c>0$, which means that $T_{1}>T_{2}$, and therefore machining along the principal direction using a ball-end cutter is more efficient. Conversely, if $\mu<\mu_{c}$ then $a \mu+b \sqrt{\mu}+c<0$ and $T_{1}<T_{2}$. Machining along the steepestslope direction using a toroidal cutter should then be privileged.

\section{A.2 Numerical analysis of the critical pa- rameter $\mu_{c}$}

The critical form factor $\mu_{c}$ can be numerically analysed like its reciprocal $s_{c}$ in Section 4.2.3. Using the same methodology presented in the previously-mentionned section, the following results are obtained. Figure 12 shows the evolution of $\mu_{c}(s)$ and the relative error of the difference between numerically calculated $T_{1}^{*}(s)$ and $T_{2}^{*}(s)$.

Like in the reciprocal case, the error is around 5\%, which confirms again the validity of the critical parameter expressions. Remark that the error is higher for small slopes, for the same reasons as the ones exposed in Section 4.2.3.

Therefore, the proposed procedure can also be carried out using the critical form factor parameter instead of the critical slope parameter. To do so, given a plane and its slope $s$, its form factor $\mu$ is compared to the critical form factor $\mu(s)$. If $\mu<\mu_{c}(s)$, then the toroidal cutter along the steepest-slope direction is selected. Otherwise, the ball-end cutter along the 

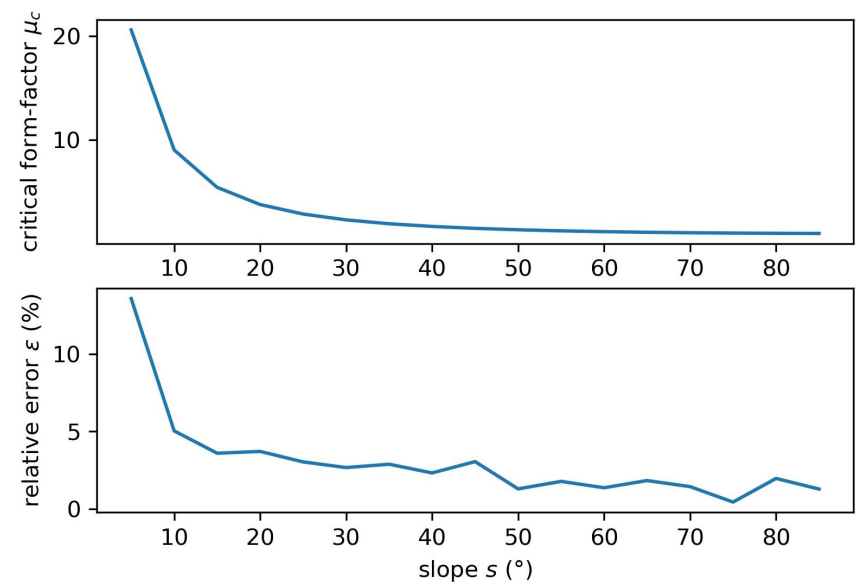

Figure 12: Evolution of critical form factor $\mu_{c}(s)$ and machining times relative error $\epsilon_{c}(s)$ with respect to slope $s$

principal direction is advised.

Remark that for both test cases presented in Sections 6.2.1 and 6.2.2, the same prediction is provided, whether a critical form factor based or a critical slope based procedure is used.

\section{B Proof that form-factor approx- imation by eigenvalues is exact for a plane}

Let $\mathbf{S}$ be a rectangular surface of width $w$ and height $h \leq w$. The coordinate system is defined so that its origin is the center of the rectangular area, axis $\mathbf{X}$ is along width direction, and axis $\mathbf{Y}$ is along height direction. Then, the coordinates of a parametric point $(u, v)$ can be written as:

$$
\mathbf{S}(u, v)=\left(\begin{array}{c}
w\left(u-\frac{1}{2}\right) \\
h\left(v-\frac{1}{2}\right) \\
0
\end{array}\right)
$$

The surface's area in the parametric space is equal to:

$$
|\mathbf{S}|=\int_{u=0}^{u=1} \int_{v=0}^{v=1} d u d v=1 .
$$

The average coordinate $S_{x}$ is then calculated:

$$
\mathrm{E}\left(S_{x}\right)=\frac{1}{|\mathbf{S}|} \int_{u=0}^{u=1} \int_{v=0}^{v=1} w\left(u-\frac{1}{2}\right) d u d v=0 .
$$

Identically, $\mathrm{E}\left(S_{y}\right)=\mathrm{E}\left(S_{z}\right)=0$. The variances are also calculated:

$$
\begin{aligned}
\operatorname{Var}\left(S_{x}\right) & =\mathrm{E}\left(S_{x}^{2}\right)-\mathrm{E}\left(S_{x}\right)^{2}=\int_{0}^{1} \int_{0}^{1} w^{2}\left(u-\frac{1}{2}\right)^{2} d u d v \\
& =\frac{w^{2}}{3}\left[\left(u-\frac{1}{2}\right)^{3}\right]_{0}^{1}=\frac{w^{2}}{12} .
\end{aligned}
$$

In the same way:

$$
\operatorname{Var}\left(S_{y}\right)=\frac{h^{2}}{12} \text { and } \operatorname{Var}\left(S_{z}\right)=0
$$

As for the covariances, they are equal to zero, since $\operatorname{Cov}\left(S_{x} S_{z}\right)=\operatorname{Cov}\left(S_{y} S_{z}\right)=0$ and:

$$
\begin{aligned}
\operatorname{Cov}\left(S_{x} S_{y}\right) & =\mathrm{E}\left(S_{x} S_{y}\right)-\mathrm{E}\left(S_{x}\right) \mathrm{E}\left(S_{y}\right) \\
& =\int_{0}^{1} \int_{0}^{1} w h\left(u-\frac{1}{2}\right)\left(v-\frac{1}{2}\right) d u d v \\
& =\int_{0}^{1} w\left(u-\frac{1}{2}\right) d u \int_{0}^{1} h\left(u-\frac{1}{2}\right) d v=0 .
\end{aligned}
$$

The covariance matrix is then:

$$
C=\left(\begin{array}{ccc}
\frac{w^{2}}{12} & 0 & 0 \\
0 & \frac{h^{2}}{12} & 0 \\
0 & 0 & 0
\end{array}\right)
$$

Because the matrix $C$ is diagonal, its eignevalues are:

$$
\lambda_{I}=\frac{w^{2}}{12} \text { and } \lambda_{I I}=\frac{h^{2}}{12}
$$

\section{Proof that the critical functions are decreasing}

The following development aims at proving that $s \mapsto \mu_{c}(s, \chi)$ and $\mu \mapsto s_{c}(\mu, \chi)$ are decreasing functions, for any value of parameter $\chi$. For the sake of simplicity, some short-hand notations are introduced: $\operatorname{sod}_{1}$, respectively $\operatorname{sod}_{2}$, is denoted $d_{1}$, respectively $d_{2}$ and $J_{\max }$ is denoted $J$. First, the derivative of step-over distance $d_{1}$ with respect to the slope angle $s$ is calculated:

$$
\frac{\partial d_{1}}{\partial s}=-2 \frac{(R-r) s h \cos s}{\sin ^{2}(s) \sqrt{2 s h\left(\frac{R-r}{\sin s}+r\right)-s^{2}}} \leq 0
$$

Then, Equation (22) can be rewritten as:

$$
\begin{aligned}
& 4 d_{2}\left(\sqrt{\frac{V}{J}}+2 \sqrt[3]{\frac{d_{1}}{2 J}}\right) \sqrt{\mu_{c}}=\frac{\sqrt{\chi}}{V}\left(d_{1}-d_{2}\right)+ \\
& \sqrt{\frac{\chi\left(d_{1}-d_{2}\right)^{2}}{V^{2}}+16 d_{2} d_{1}\left(\sqrt{\frac{V}{J}}+2 \sqrt[3]{\frac{d_{1}}{2 J}}\right)\left(\sqrt{\frac{V}{J}}+2 \sqrt[3]{\frac{d_{2}}{2 J}}\right)}
\end{aligned}
$$

The last equation is differentiated with respect to $d_{1}$ to obtain:

$$
\begin{aligned}
& 4 d_{2}\left(\sqrt{\frac{V}{J}}+2 \sqrt[3]{\frac{d_{1}}{2 J}}\right) \frac{\partial \sqrt{\mu_{c}}}{\partial d_{1}}+4 d_{2}\left(\sqrt{\frac{V}{J}}+\frac{2}{3} \sqrt[3]{\frac{1}{2 J d_{1}^{2}}}\right) \sqrt{\mu_{c}}= \\
& \frac{\sqrt{\chi}}{V}+\frac{\frac{\chi\left(d_{1}-d_{2}\right)}{V^{2}}+8 d_{2}\left(\sqrt{\frac{V}{J}}+2 \sqrt[3]{\frac{d_{2}}{2 J}}\right)\left(\sqrt{\frac{V}{J}}+\frac{8}{3} \sqrt[3]{\frac{d_{1}}{2 J}}\right)}{\sqrt{\frac{\chi\left(d_{1}-d_{2}\right)^{2}}{V^{2}}+16 d_{1} d_{2}\left(\sqrt{\frac{V}{J}}+2 \sqrt[3]{\frac{d_{1}}{2 J}}\right)\left(\sqrt{\frac{V}{J}}+2 \sqrt[3]{\frac{d_{2}}{2 J}}\right)}}
\end{aligned}
$$

The second term of the right-hand side (the big fraction) is positive. Therefore, one easily obtain:

$$
\frac{4 d_{2}}{\sqrt{\mu_{c}}}\left(\sqrt{\frac{V}{J}}+2 \sqrt[3]{\frac{d_{1}}{2 J}}\right) \frac{\partial \sqrt{\mu_{c}}}{\partial d_{1}} \geq \frac{\sqrt{\chi}}{V \sqrt{\mu_{c}}}-4 d_{2}\left(\sqrt{\frac{V}{J}}+\frac{2}{3} \sqrt[3]{\frac{1}{2 J d_{1}^{2}}}\right)
$$


Let $w_{c}$, respectively $h_{c}$, be the critical width, respectively the height at critical form-factor $\mu_{c}(s, \chi)$. Thus:

$$
\sqrt{\frac{\chi}{\mu_{c}}}=\sqrt{w_{c} h_{c} \frac{h_{c}}{w_{c}}}=h_{c}
$$

Besides:

$$
\frac{4 d_{2}}{\sqrt{\mu_{c}}}\left(\sqrt{\frac{V}{J}}+2 \sqrt[3]{\frac{d_{1}}{2 J}}\right) \geq 0
$$

As a result $\frac{\partial \sqrt{\mu_{c}}}{\partial d_{1}}$, is positive if:

$$
h_{c} \geq 4 d_{2} V\left(\sqrt{\frac{V}{J}}+\frac{2}{3} \sqrt[3]{\frac{1}{2 J d_{1}^{2}}}\right)
$$

The value of $d_{1}$ depends on the slope $s$ and is minimal when $d_{1}=d_{2}$, in the worst case (in the sense of the previous inequality). Thus, with the numerical values used for parameters $R$, $r, J, V$ and $s h, h_{c} \geq 5.3 \mathrm{~mm}$ is a condition sufficient to ensure that the derivative of $\sqrt{\mu_{c}}$ with respect to $d_{1}$ is positive. Varying tool and machining parameters in ranges of realistic values lead to an inferior bound on $h_{c}$ varying between $3 \mathrm{~mm}$ and $10 \mathrm{~mm}$. However, such values are very small for the dimensions of surfaces to be machined in practice, therefore:

$$
\frac{\partial \mu_{c}}{\partial s}=\frac{\partial \mu_{c}}{\partial \sqrt{\mu_{c}}} \frac{\partial \sqrt{\mu_{c}}}{\partial d_{1}} \frac{\partial d_{1}}{\partial s}=2 \sqrt{\mu_{c}} \frac{\partial \sqrt{\mu_{c}}}{\partial d_{1}} \frac{\partial d_{1}}{\partial s} \leq 0 .
$$

In conclusion, $s \mapsto \mu_{c}(s, \chi)$ is a decreasing function. The function $\mu \mapsto s_{c}(\mu, \chi)$ is also decreasing since it is the reciprocal function of $s \mapsto \mu_{c}(s, \chi)$.

\section{Proof that the critical slope in- creases with $\chi$}

The considerations presented in Section 7.4 state that the critical parameter $s_{c}(\mu)$ increases with $\chi$ value. This statement is proven here, first by proving that $\mu_{c}(s)$ increases with $\chi$, and then by using the fact that $\mu_{c}(s)$ and $s_{c}(\mu)$ are reciprocal functions.

To prove that $\mu_{c}(s)$ increases with $\chi$, the derivative of $\mu_{c}$ with respect to $\chi$ (denoted $\frac{\partial \mu_{c}}{\partial \chi}$ ) has to be calculated first, all other parameters being fixed. Equation (23), gives the expression of the derivative $\frac{\partial \mu_{c}}{\partial \chi}$ calculated from its analytical expression (Equation (22)):

$$
\frac{\partial \mu_{c}}{\partial \chi}=\frac{\mu_{c}}{\beta_{1}} \frac{\beta_{2}}{2 \sqrt{\chi}}+\frac{\beta_{2}^{2}}{2 \sqrt{\chi \beta_{2}^{2}+4 \beta_{1} \beta_{3}}},
$$

$$
\text { where } \begin{aligned}
\beta_{1} & =2 \frac{\operatorname{sod}_{2}}{\operatorname{sod}_{1}}\left(\sqrt{\frac{V}{J_{\max }}}+2 \sqrt[3]{\frac{\operatorname{sod}_{1}}{2 J_{\max }}}\right) \geq 0 \\
\beta_{2} & =\frac{\left(1-\frac{\text { sod }_{2}}{\operatorname{sod}_{1}}\right)}{V} \geq 0 \\
\beta_{3} & =2\left(\sqrt{\frac{V}{J_{\max }}}+2 \sqrt[3]{\frac{\operatorname{sod}_{2}}{2 J_{\max }}}\right) \geq 0 .
\end{aligned}
$$

It is clear from this expression that the derivative is positive. Thus $\mu_{c}$ increases with $\chi$.

Let $s \in[0, \pi / 2]$ and $\mu \geq 1$. From the definition of the critical parameters, it is straigthforward that for any value of parameter $\chi$ constant: $\mu_{c}(s)$ is the value that the form factor should take if the slope is equal to $s$, so that machining times in both cases are equal. Consequently, if the value of $\mu$ is equal to $\mu_{c}(s)$, then the corresponding critical slope would be equal to $s$. In other words, $\mu_{c}$ and $s_{c}$ are reciprocal functions:

$$
\forall s \in\left[0, \frac{\pi}{2}\right] s_{c}\left(\mu_{c}(s)\right)=s \text { and } \forall \mu \geq 1 \mu_{c}\left(s_{c}(\mu)\right)=\mu .
$$

The first statement can be re-written including the dependency on $\chi$ :

$$
\forall s \in\left[0, \frac{\pi}{2}\right], \forall \chi \geq 0, s_{c}\left(\mu_{c}(s, \chi), \chi\right)=s .
$$

Deriving the previous equation with respect to $\chi$ for constant $s$, using the total derivative formula leads to:

$$
\frac{\partial s_{c}}{\partial \chi}\left(\mu_{c}(s, \chi), \chi\right)=-\frac{\partial \mu_{c}}{\partial \chi}(s, \chi) \frac{\partial s_{c}}{\partial \mu}\left(\mu_{c}(s, \chi), \chi\right)
$$

It was just shown that $\frac{\partial \mu_{c}}{\partial \chi}$ is positive. In addition, it has be proven that $s_{c}$ is a decreasing function of $\mu$ (see Appendix C). Thus, $\frac{\partial s_{c}}{\partial \mu}$ is negative. Since $\mu_{c}$ is a bijection, for each $\mu \geq 1$ there exists (a unique) $s \in[0, \pi / 2]$ such as $\mu=\mu_{c}(s, \chi)$. Therefore, the term $\frac{\partial s_{c}}{\partial \chi}(\mu, \chi)$ is positive for each value of $\mu$, and $s_{c}$ is an increasing function of $\chi$. 